\title{
Area distribution and scaling function for punctured polygons
}

\author{
Christoph Richard $\dagger$, Iwan Jensen $\ddagger$, Anthony J. Guttmann $\ddagger$ \\ $\dagger$ Fakultät für Mathematik, Universität Bielefeld, \\ Postfach 1001 31, 33501 Bielefeld, Germany \\ richard@math.uni-bielefeld.de
}

$\ddagger$ ARC Centre of Excellence for Mathematics and Statistics of Complex Systems, Department of Mathematics and Statistics, The University of Melbourne,

Victoria 3010, Australia

$\{$ I. Jensen, tonyg\}@ms . unimelb.edu. au

Submitted: Jan 22, 2007; Accepted: Apr 5, 2008; Published: Apr 10, 2008

Mathematics Subject Classifications: 05A15, 05A16

\begin{abstract}
Punctured polygons are polygons with internal holes which are also polygons. The external and internal polygons are of the same type, and they are mutually as well as self-avoiding. Based on an assumption about the limiting area distribution for unpunctured polygons, we rigorously analyse the effect of a finite number of punctures on the limiting area distribution in a uniform ensemble, where punctured polygons with equal perimeter have the same probability of occurrence. Our analysis leads to conjectures about the scaling behaviour of the models.

We also analyse exact enumeration data. For staircase polygons with punctures of fixed size, this yields explicit expressions for the generating functions of the first few area moments. For staircase polygons with punctures of arbitrary size, a careful numerical analysis yields very accurate estimates for the area moments. Interestingly, we find that the leading correction term for each area moment is proportional to the corresponding area moment with one less puncture. We finally analyse corresponding quantities for punctured self-avoiding polygons and find agreement with the conjectured formulas to at least 3-4 significant digits.
\end{abstract}

\section{Introduction}

The behaviour of planar self-avoiding walks (SAW) and polygons (SAP) is one of the classical unsolved problems, not only of algebraic combinatorics, but also of chemistry and 
of physics $[1,2,3]$. In the field of algebraic combinatorics, it is a classical enumeration problem. In chemistry and physics, SAWs and SAPs are used to model a variety of phenomena, including the properties of long-chain polymers in dilute solution [4], the behaviour of ring polymers and vesicles in general [5] and benzenoid systems [6, 7] in particular. Though the qualitative form of the phase diagram [8] is known rigorously, there is otherwise a paucity of rigorous results. However, there are a few conjectures, including the exact values of the critical exponents $[9,10]$, and more recently the limit distribution of area and scaling function for SAPs, when enumerated by both area and perimeter $[11,12,13,14,15]$.

Models of planar polygons with punctures arise naturally as cross-sections of threedimensional vesicle models. In such cross-sections, there may be holes within holes, and the number of punctures may be infinite. In this work, we exclude these possibilities. Whereas our methods can be used to study the former case, the second situation presents new difficulties, which we have not yet overcome ${ }^{1}$.

In this work we consider the effect of a finite number of punctures in polygon models, in particular we study staircase polygons and self-avoiding polygons on the square lattice. The perimeter of a punctured polygon $[16,17]$ is the perimeter of its boundary (both internal and external) while the area of a punctured polygon is the area of the enclosed by the external perimeter minus the area(s) of any holes ${ }^{2}$. As discussed in section 2 below, the effect of punctures on the critical point and critical exponents of the area and perimeter generating function has been the subject of previous studies, but the effect of punctures on the critical amplitudes and detailed asymptotics have not, to our knowledge, been previously considered.

Apart from the intrinsic interest of the problem, we also believe it to be the appropriate route to study the detailed asymptotics of polyominoes, since punctured polygons are a subclass of polyominoes. While we still have some way to go to understand the polyomino phase diagram, we feel that restricting the problem to this important subclass is the correct route.

The make-up of the paper is as follows: In the next section we review the known situation for the perimeter and area generating functions of punctured polygons and polyominoes. In section 3 we review the phase diagram and scaling behaviour of staircase polygons and self-avoiding polygons. In section 4 we rigorously express the asymptotic behaviour of models of punctured polygons in the limit of large perimeter in terms of the asymptotic behaviour of the model without punctures, by refining arguments used in [16]. This leads, in particular, to a characterisation of the limit distribution of the area of punctured polygons. This result is then used to conjecture scaling functions of punctured

\footnotetext{
${ }^{1}$ Since punctured polygons with an unlimited number of punctures have, in contrast to polygons without punctures, an (ordinary) perimeter generating function with zero radius of convergence [18], both the phase diagram and the detailed asymptotics are clearly going to be very different from those of polygons without punctures. This is discussed further in the conclusion.

${ }^{2}$ This has to be distinguished from so-called composite polygons [19]. The perimeter of a composite polygon is defined as the perimeter of the external polygon only, resulting in asymptotic behaviour different from punctured polygons. Moreover, composite polygons can have more complex internal structure than just other polygons.
} 
polygons. We consider three cases of increasing generality. First, we consider the case of minimal punctures. It is shown that effects of self-avoidance are asymptotically irrelevant, and that elementary area counting arguments yield the leading asymptotic behaviour. We then discuss the case of a finite number of punctures of bounded size, and finally the case of a finite number of punctures of unbounded size. Results for the latter case are given for models with a finite critical perimeter generating function such as staircase polygons and self-avoiding polygons. Whereas the latter two cases are technically more involved, the underlying arguments are similar to the case of minimal punctures. If the critical perimeter generating function of the polygon model without punctures is finite, then all three cases lead, up to normalisation, to the same limit distributions and scaling function conjectures.

The next two sections discuss the development and application of extensive numerical data to test the results of the previous section. Moreover, the numerical analysis yields predictions, conjectured to be exact, for the corrections to the asymptotic behaviour. In particular, section 5 describes the very efficient algorithms used to generate the data, while section 6 applies a range of numerical tools to the analysis of the generating functions for punctured staircase polygons and then punctured self-avoiding polygons. Here we wish to emphasise that our work on this problem involved a close interplay between analytical and numerical work. Initially, our intention was to check our predictions for scaling functions by studying amplitude ratios for area moments (given in Table 1). We subsequently discovered numerically the exact solutions for minimally punctured staircase polygons. We also obtained very accurate estimates for the amplitudes of staircase polygons with one or two punctures of arbitrary size. From these results we were able to conjecture exact expressions for the amplitudes, which in turn spurred us on to further analytical work in order to prove these results. The final section summarises and discusses our results.

\section{Punctured polygons}

We consider polygons on the square lattice in this article. In particular, we study selfavoiding polygons and staircase polygons. A self-avoiding polygon on a lattice can be defined as a walk along the edges of the lattice, which starts and ends at the same lattice point, but has no other self-intersections. When counting SAPs, they are generally considered distinct up to translations, change of starting point, and orientation of the walk, so if there are $p_{m}$ SAPs of length or perimeter $m$ there are $2 m p_{m}$ walks (the factor of two arising since the walk can go in two directions). On the square lattice the perimeter of any polygon is always even so it is natural to count polygons by half-perimeter instead of perimeter. The area of a polygon is the number of lattice cells (times the area of the unit cell) enclosed by the perimeter of the polygon. A (square lattice) staircase polygon can be defined as the intersection of two mutually avoiding directed walks starting at the same lattice point, moving only to the right or up and terminating once the walks join at a vertex. Every staircase polygon is a self-avoiding polygon. It is well known that the number $p_{m}$ of staircase polygons of half-perimeter $m$ is given by the $(m-1)^{t h}$ Catalan 


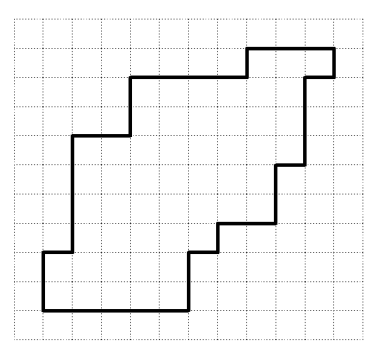

Staircase Polygon

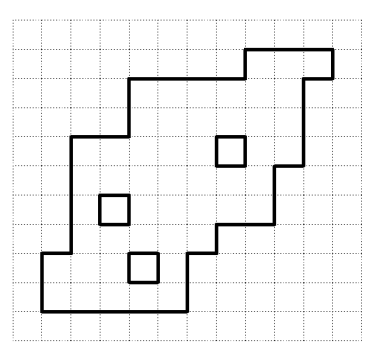

Minimally Punctured Staircase

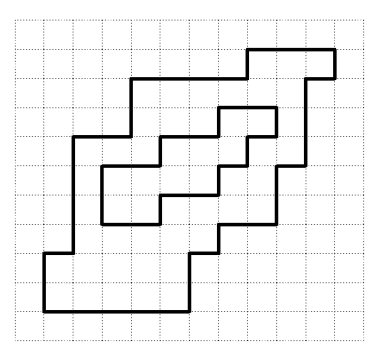

Once Punctured Staircase

Figure 1: Examples of the types of staircase polygons we consider in this paper.

number, $p_{m}=\left(\begin{array}{c}2 m-2 \\ m-1\end{array}\right) / m$, with half-perimeter generating function

$$
\mathcal{P}(x)=\sum_{m} p_{m} x^{m}=\frac{1-2 x-\sqrt{1-4 x}}{2} \sim \frac{1}{4}-\frac{1}{2}(1-\mu x)^{2-\alpha} \quad(\mu x \nearrow 1),
$$

where the connective constant $\mu=4$ and the critical exponent $\alpha=3 / 2$. Recall that $f(x) \sim g(x)$ as $x \nearrow x_{c}$ means that $\lim f(x) / g(x)=1$ as $x \rightarrow x_{c}$ from below. In addition, as usual, the rhs is understood as the first two leading terms in an asymptotic expansion of the lhs about $x=1 / \mu$, see e.g. [20, Sec 1].

Punctured polygons [16] are polygons with internal holes which are also polygons (the polygons are mutually- as well as self-avoiding). The perimeter of a punctured polygon is the sum of the external and internal perimeters while the area is the area of the external polygon minus the areas of the internal polygons. We also consider polygons with minimal punctures, that is, polygons where the punctures are unit cells (or polygons with perimeter 4 and area 1). Punctured staircase polygons are illustrated in figure 1.

We briefly review the situation for SAPs with punctures. Analogous results can be shown to hold for staircase polygons with punctures. Square lattice SAPs with $r$ punctures, counted by area $n$, were first studied by Janse van Rensburg and Whittington [17]. They proved the existence of an exponential growth constant $\kappa^{(r)}$ satisfying $\kappa^{(r)}=\kappa^{(0)}=\kappa$. Denoting the corresponding number of SAPs by $a_{n}^{(r)}$ and assuming asymptotic behaviour of the form

$$
a_{n}^{(r)} \sim A^{(r)}\left(\kappa^{(r)}\right)^{n} n^{\beta_{r}-1} \quad(n \rightarrow \infty),
$$

Janse van Rensburg proved [21] that $\beta_{r}=\beta_{0}+r$. These results of course translate to the singular behaviour of the corresponding generating functions, defined by $\mathcal{A}^{(r)}(q)=$ $\sum_{n>0} a_{n}^{(r)} q^{n}$.

In [16] Guttmann, Jensen, Wong and Enting studied square lattice SAPs with $r$ punctures counted by half-perimeter $m$. They proved the existence of an exponential growth constant $\mu^{(r)}$ satisfying $\mu^{(r)}=\mu^{(0)}=\mu$. If the corresponding number $p_{m}^{(r)}$ of SAPs is assumed to behave asymptotically as

$$
p_{m}^{(r)} \sim B^{(r)}\left(\mu^{(r)}\right)^{m} m^{\alpha_{r}-3} \quad(m \rightarrow \infty),
$$


they argued, on the basis of a non-rigorous argument, that $\alpha_{r}=\alpha_{0}+\frac{3}{2} r$. Their results also translate to the associated half-perimeter generating function $\mathcal{P}^{(r)}(x)=\sum_{m>0} p_{m}^{(r)} x^{m}$ correspondingly.

Similar results were obtained for polyominoes enumerated by number of cells (i.e. area) with a finite number $r$ of punctures [16]. It has been proved that an exponential growth constant $\tau$ exists independently of $r$, which satisfies $4.06258 \approx \tau>\kappa \approx 3.97087$, where $\kappa$ is the growth constant for SAPs enumerated by area. If the number $a_{n}^{(r)}$ of polynominoes of area $n$ with $r$ punctures is assumed to satisfy asymptotically

$$
a_{n}^{(r)} \sim C^{(r)}\left(\tau^{(r)}\right)^{n} n^{\gamma_{r}-1} \quad(n \rightarrow \infty),
$$

it has been shown that $\gamma_{r}=\gamma_{0}+r$ and hence that, if the exponents $\gamma_{r}$ exist, they increase by 1 per puncture. It was further conjectured on the basis of extensive numerical studies [16], that the number $a_{n}^{(r)}$ satisfies asymptotically

$$
a_{n}^{(r)} \sim \tau^{n} n^{r-1} \sum_{i \geq 0} C_{i}^{(r)} / n^{i} \quad(n \rightarrow \infty) .
$$

Notice the conjecture $\gamma_{0}=0$ and that the correction terms go down by a whole power.

For unrestricted polyominoes, that is to say, with no restriction on the number of punctures, it was proved by Guttmann, Jensen and Owczarek [18] that the perimeter generating function has zero radius of convergence. The perimeter is defined to be the perimeter of the boundary plus the total perimeter of any holes. If $p_{m}$ denotes the number of polyominoes, distinct up to a translation, with half-perimeter $m$, they proved that $p_{m}=m^{m / 4+o(m)}$, meaning that

$$
\lim _{m \rightarrow \infty} \frac{\log p_{m}}{m \log m}=\frac{1}{4}
$$

An attempt to study the quasi-exponential generating function with coefficients $r_{m}=$ $p_{m} / \Gamma(m / 4+1)$ was equivocal. For that reason, studying punctured self-avoiding polygons was considered a controlled route to attempt to determine the two-variable area-perimeter generating function of polyominoes.

In passing, we note that in [22] the exact solution of the perimeter generating function for staircase polygons with a staircase hole is conjectured, in the form of an $8^{\text {th }}$ order ODE. It is not obvious how to extract particular asymptotic information, notably critical amplitudes from the solution without numerically integrating the ODE. In the following, we will obtain such information by combinatorial arguments, which refine those of [16].

\section{Polygon models and their scaling behaviour}

We review the asymptotic behaviour of self-avoiding polygons and staircase polygons following mainly [8]. For concreteness, consider the fixed perimeter ensemble where, for fixed half-perimeter $m$, each polygon of area $n$ has a weight proportional to $q^{n}$, for 
some positive real number $q$. If $0<q<1$, polygons of large area are exponentially suppressed, so that typical polygons should be ramified objects. Since such polygons would closely resemble branched polymers, the phase $0<q<1$ is also referred to as the branched polymer phase. As $q$ approaches unity, typical polygons should fill out more, and become less string-like. For $q>1$, polygons of small area are exponentially suppressed, so that typical polygons should become "fat". Indeed, they resemble convex polygons [23] and it has been proved [8] that the mean area of polygons of half-perimeter $m$ grows asymptotically proportional to $m^{2}$. In the extended phase $q=1$, it is numerically very well established that the mean area of polygons of half-perimeter $m$ grows asymptotically proportionally to $m^{3 / 2}$. In the branched polymer phase $0<q<1$, the mean area of polygons of half-perimeter $m$ is expected to grow asymptotically linearly in $m$, compare also [24, Thm 7.6] and [25, Ch IX.6, Ex. 12].

This change of asymptotic behaviour of typical polygons w.r.t. $q$ is reflected in the singular behaviour of the half-perimeter and area generating function

$$
\mathcal{P}(x, q)=\sum_{m, n} p_{m, n} x^{m} q^{n},
$$

where $p_{m, n}$ denotes the number of (self-avoiding) polygons of half-perimeter $m$ and area $n$. It has been proved [8] that the free energy

$$
\kappa(q):=\lim _{m \rightarrow \infty} \frac{1}{m} \log \left(\sum_{n} p_{m, n} q^{n}\right)
$$

exists and is finite if $0<q \leq 1$. Further, $\kappa(q)$ is log-convex and continuous for these values of $q$. It is infinite for $q>1$. It was proved that for fixed $0<q \leq 1$, the radius of convergence $x_{c}(q)$ of $\mathcal{P}(x, q)$ is given by $x_{c}(q)=e^{-\kappa(q)}$. For fixed $q>1, \mathcal{P}(x, q)$ has zero radius of convergence. Fisher et al. [8] obtained rigorous upper and lower bounds on $x_{c}(q)$. The expected phase diagram, i.e., the radius of convergence of $\mathcal{P}(x, q)$ in the $x-q$ plane, as estimated numerically from extrapolation of SAP enumeration data by perimeter and area, is sketched qualitatively in figure 2 .

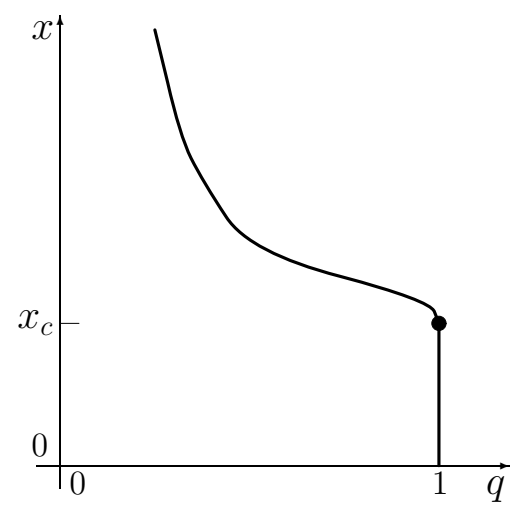

Figure 2: A sketch of the phase diagram of self-avoiding polygons. 
For $0<q<1$, the line $x_{c}(q)$ is, for self-avoiding polygons, expected to be a line of logarithmic singularities of the generating function $\mathcal{P}(x, q)$. For branched polymers in the continuum limit, the existence of the logarithmic singularity has recently been proved [26]. The line $q=1$ is, for $0<x<x_{c}:=x_{c}(1)$, a line of finite essential singularities [8]. For staircase polygons, counted by half-perimeter and area, the corresponding phase diagram can be determined exactly, and is qualitatively similar to that of self-avoiding polygons. Along the line $x_{c}(q)$ the half-perimeter and area generating function diverges with a simple pole, and the line $q=1$ is, for $0<x<x_{c}$, a line of finite essential singularities [27].

We will focus on the uniform fixed perimeter ensemble $q=1$ in this article. Whereas asymptotic area laws in the fixed perimeter ensemble are expected to be Gaussian for positive $q \neq 1$, the behaviour in the uniform fixed perimeter ensemble $q=1$ is more interesting. For staircase polygons, it can be shown that a limit distribution of area exists and is given by the Airy distribution [28, 29, 30]. For self-avoiding polygons, it is conjectured that an area limit law exists and is given by the Airy distribution, on the basis of a detailed numerical analysis $[11,14,15]$. See subsections 4.1 and 4.4.

If $p_{m, n}$ denotes the number of polygons of half-perimeter $m$ and area $n$, the existence and the form of a limit distribution can be inferred from the asymptotic behaviour of the factorial moment coefficients $\sum_{n}(n)_{k} p_{m, n}$, where $(a)_{k}=a \cdot(a-1) \cdot \ldots \cdot(a-k+1)$. The following result is obtained by standard reasoning [31].

Proposition 1. Let for $m, n \in \mathbb{N}_{0}$ real numbers $p_{m, n}$ be given. Assume that the numbers $p_{m, n}$ have the asymptotic form, for $k \in \mathbb{N}_{0}$,

$$
\sum_{n}(n)_{k} p_{m, n} \sim A_{k} x_{c}^{-m} m^{\gamma_{k}-1} \quad(m \rightarrow \infty)
$$

for positive real numbers $A_{k}$ and $x_{c}$, where $\gamma_{k}=(k-\theta) / \phi$, with real constants $\theta$ and $\phi>0$. Assume that the numbers $M_{k}:=A_{k} / A_{0}$ satisfy the Carleman condition

$$
\sum_{k=0}^{\infty}\left(M_{2 k}\right)^{-1 / 2 k}=+\infty .
$$

Then, for almost all $m$, the random variables $\widetilde{X}_{m}$ of area in the uniform fixed perimeter ensemble

$$
\mathbb{P}\left(\widetilde{X}_{m}=n\right)=\frac{p_{m, n}}{\sum_{n} p_{m, n}}
$$

are well defined. We have

$$
X_{m}:=\frac{\widetilde{X}_{m}}{m^{1 / \phi}} \stackrel{d}{\longrightarrow} X \quad(m \rightarrow \infty),
$$

for a uniquely defined random variable $X$ with moments $M_{k}$, where the superscript $d$ denotes convergence in distribution. We also have moment convergence. 
Sketch of proof. A straightforward calculation using Eq. (2) leads to

$$
\mathbb{E}\left[\left(\widetilde{X}_{m}\right)_{k}\right] \sim \frac{A_{k}}{A_{0}} m^{k / \phi} \quad(m \rightarrow \infty) .
$$

It follows that asymptotically the factorial moments are equal to the (ordinary) moments. Thus, the moments of $X_{m}$ have the same asymptotic form

$$
\mathbb{E}\left[\left(X_{m}\right)^{k}\right] \sim \frac{A_{k}}{A_{0}}=M_{k} \quad(m \rightarrow \infty) .
$$

Due to the growth condition Eq. (3), the sequence $\left(M_{k}\right)_{k \in \mathbb{N}_{0}}$ defines a unique random variable $X$ with moments $M_{k}$. Moment convergence of $\left(X_{m}\right)$ implies convergence in distribution, see [31, Thm 4.5.5] for the line of arguments.

The assumption Eq. (2) translates, on the level of the half-perimeter and area generating function $\mathcal{P}(x, q)$, to a certain asymptotic behaviour of the so-called factorial moment generating functions

$$
g_{k}(x)=\left.\frac{(-1)^{k}}{k !} \frac{\partial^{k}}{\partial q^{k}} \mathcal{P}(x, q)\right|_{q=1} .
$$

It can be shown (compare [32]) that the asymptotic behaviour Eq. (2) implies for $\gamma_{k}>0$ the asymptotic equivalence

$$
g_{k}(x) \sim \frac{f_{k}}{\left(x_{c}-x\right)^{\gamma_{k}}} \quad\left(x \nearrow x_{c}\right),
$$

where the amplitudes $f_{k}$ are related to the amplitudes $A_{k}^{3}$ in Proposition 1 via

$$
f_{k}=\frac{(-1)^{k}}{k !} A_{k} x_{c}^{\gamma_{k}} \Gamma\left(\gamma_{k}\right) .
$$

If $-1<\gamma_{k}<0$, the series $g_{k}(x)$ is convergent as $x \nearrow x_{c}$, and the same estimate Eq. (4) holds, with $g_{k}(x)$ replaced by $g_{k}(x)-g_{k}\left(x_{c}\right)$, where $g\left(x_{c}\right):=\lim _{x \succ x_{c}} g(x)$. In order to deal with these two different cases, we define for a power series $g(x)$ with radius of convergence $x_{c}$, the number

$$
g^{(c)}=\left\{\begin{array}{cc}
g\left(x_{c}\right) & \text { if }\left|\lim _{x / x_{c}} g(x)\right|<\infty \\
0 & \text { otherwise. }
\end{array}\right.
$$

Adopting the generating function point of view, the amplitudes $f_{k}$ determine the numbers $A_{k}$ and hence the moments $M_{k}=A_{k} / A_{0}$ of the limit distribution. The formal series $F(s)=\sum_{k \geq 0} f_{k} s^{-\gamma_{k}}$ will appear frequently in the sequel.

\footnotetext{
${ }^{3}$ Note that our definition of the amplitudes $A_{k}$ differs from that in [13] by a factor of $(-1)^{k} k$ ! and from that in $[33,34]$ by a factor of $k$ !.
} 
Definition 1. For the generating function $\mathcal{P}(x, q)$ of a class of self-avoiding polygons, denote its factorial moment generating functions by

$$
g_{k}(x)=\left.\frac{(-1)^{k}}{k !} \frac{\partial^{k}}{\partial q^{k}} \mathcal{P}(x, q)\right|_{q=1} .
$$

Assume that the factorial moment generating functions satisfy

$$
g_{k}(x)-g_{k}^{(c)} \sim \frac{f_{k}}{\left(x_{c}-x\right)^{\gamma_{k}}} \quad\left(x \nearrow x_{c}\right),
$$

with real exponents $\gamma_{k}$. Then, the formal series

$$
F(s)=\sum_{k \geq 0} f_{k} s^{-\gamma_{k}}
$$

is called the area amplitude series.

The area amplitude series is expected to approximate the half-perimeter and area generating function $\mathcal{P}(x, q)$ about $(x, q)=\left(x_{c}, 1\right)$. This is motivated by the following heuristic argument. Assume that $\gamma_{k}=(k-\theta) / \phi$ with $\phi>0$ and argue

$$
\begin{aligned}
\mathcal{P}(x, q) & \approx \sum_{k \geq 0}\left(g_{k}^{(c)}+\frac{f_{k}}{\left(x_{c}-x\right)^{\gamma_{k}}}\right)(1-q)^{k} \\
& \approx\left(\sum_{k \geq 0} g_{k}^{(c)}(1-q)^{k}\right)+(1-q)^{\theta}\left(\sum_{k \geq 0} f_{k}\left(\frac{x_{c}-x}{(1-q)^{\phi}}\right)^{-\gamma_{k}}\right) .
\end{aligned}
$$

In the above calculation, we formally expanded $\mathcal{P}(x, q)$ about $q=1$ and then replaced the Taylor coefficients by their leading singular behaviour about $x=x_{c}$. In the rhs of the above expression, the first sum is by assumption finite, and the second term contains the area amplitude series $F(s)$ of combined argument $s=\left(x_{c}-x\right) /(1-q)^{\phi}$. This motivates the following definition. A class of self-avoiding polygons is a subset of self-avoiding polygons. Prominent examples are, among others [35], self-avoiding polygons and staircase polygons.

Definition 2. Let a class of square lattice self-avoiding polygons be given, with halfperimeter and area generating function $\mathcal{P}(x, q)$. Let $0<x_{c}<\infty$ be the radius of convergence of the half-perimeter generating function $\mathcal{P}(x, 1)$. Assume that there exist a constant $s_{0} \in[-\infty, 0)$, a function $\mathcal{F}:\left(s_{0}, \infty\right) \rightarrow \mathbb{R}$, a real constant $A$ and real numbers $\theta$ and $\phi>0$, such that the generating function $\mathcal{P}(x, q)$ satisfies, for real $x$ and $q$, where $0<q<1$ and $\left(x_{c}-x\right) /(1-q)^{\phi} \in\left(s_{0}, \infty\right)$, the asymptotic equivalence

$$
\mathcal{P}(x, q)-A \sim(1-q)^{\theta} \mathcal{F}\left(\frac{x_{c}-x}{(1-q)^{\phi}}\right) \quad(x, q) \longrightarrow\left(x_{c}, 1\right) .
$$

Then, the function $\mathcal{F}(s)$ is called a scaling function of combined argument $s=\left(x_{c}-\right.$ $x) /(1-q)^{\phi}$, and $\theta$ and $\phi$ are called critical exponents. 
Remarks. i) Due to the restriction on the argument of the scaling function, the limit $(x, q) \rightarrow\left(x_{c}, 1\right)$ is approached for values $(x, q)$ satisfying $x<x_{0}(q)$ and $q<1$, where $x_{0}(q)=x_{c}-s_{0}(1-q)^{\phi}$.

ii) The above scaling form is also suggested by the theory of tricritical scaling, adapted to polygon models [36]. The scaling function describes the leading singular behaviour of $\mathcal{P}(x, q)$ about the point $\left(x_{c}, 1\right)$ where the two lines of qualitatively different singularities meet.

iii) The additional condition $\phi>\theta$ and $\theta \notin \mathbb{N}_{0}$ ensure that $\gamma_{k} \in(-1, \infty) \backslash\{0\}$. Then, by the above argument, it is plausible that there exists an asymptotic expansion of the scaling function $\mathcal{F}(s)$ about infinity coinciding with the area amplitude series $F(s)$, i.e., $\mathcal{F}(s) \sim F(s)$ as $s \rightarrow \infty$. Recall that $s$ is considered to be a real parameter.

For staircase polygons the existence of a scaling form Eq. (7) has been proved [27, Thm 5.3], with scaling function $\mathcal{F}(s):\left(s_{0}, \infty\right) \rightarrow \mathbb{R}$ explicitly given by

$$
\mathcal{F}(s)=\frac{1}{16} \frac{\mathrm{d}}{\mathrm{d} s} \log \operatorname{Ai}\left(2^{8 / 3} s\right),
$$

with exponents $\theta=1 / 3$ and $\phi=2 / 3$ and $x_{c}=1 / 4$, where $\operatorname{Ai}(x)=\frac{1}{\pi} \int_{0}^{\infty} \cos \left(t^{3} / 3+\right.$ $t x) \mathrm{d} t$ is the Airy function. The constant $s_{0}$ is such that $2^{8 / 3} s_{0}$ is the location of the Airy function zero of smallest modulus. For rooted SAPs with half-perimeter and area generating function $\mathcal{P}_{r}(x, q)=x \frac{\mathrm{d}}{\mathrm{d} x} \mathcal{P}(x, q)$, the conjectured form of the scaling function $\mathcal{F}_{r}(s):\left(s_{0}, \infty\right) \rightarrow \mathbb{R}$ is $[13]$

$$
\mathcal{F}_{r}(s)=\frac{x_{c}}{2 \pi} \frac{\mathrm{d}}{\mathrm{d} s} \log \operatorname{Ai}\left(\frac{\pi}{x_{c}}\left(4 A_{0}\right)^{\frac{2}{3}} s\right),
$$

with the same exponents as for staircase polygons, $\theta=1 / 3$ and $\phi=2 / 3$. Here, $x_{c}=$ $0.14368062927(2)$ is the radius of convergence of the half-perimeter generating function $\mathcal{P}_{r}(x, 1)$ of (rooted) SAPs, and $A_{0}=0.09940174(4)$ is the critical amplitude $\sum_{n} m p_{m, n} \sim$ $A_{0} x_{c}^{-m} m^{-3 / 2}$ of rooted SAPs, which coincides with the critical amplitude $A_{0}$ of (unrooted) SAPs. Again, the constant $s_{0}$ is such that the corresponding Airy function argument is the location of the Airy function zero of smallest modulus. This conjecture was based on the conjecture that both models have, up to normalisation constants, the same area amplitude series. The latter conjecture is supported numerically to very high accuracy by an extrapolation of the moment series using exact enumeration data [11, 14]. The conjectured form of the scaling function $\mathcal{F}(s):\left(s_{0}, \infty\right) \rightarrow \mathbb{R}$ for SAPs is obtained by integration,

$$
\mathcal{F}(s)=-\frac{1}{2 \pi} \log \operatorname{Ai}\left(\frac{\pi}{x_{c}}\left(4 A_{0}\right)^{\frac{2}{3}} s\right)+C(q),
$$

with exponents $\theta=1$ and $\phi=2 / 3$. In the above formula, $C(q)$ is a $q$ dependent constant of integration, $C(q)=\frac{1}{12 \pi}(1-q) \log (1-q)$, see [15]. Corresponding results for the triangular and hexagonal lattices can be found in [11].

For models of punctured polygons with a finite number of punctures, we have qualitatively the same phase diagram as for polygon models without punctures, however with 
different critical exponents $\theta$ depending on the number of punctures $[21,16]$, and hence we expect different scaling functions. We will focus on critical exponents and area limit laws in the uniform ensemble $q=1$ in the following section. This will lead to conjectures for the corresponding scaling functions.

\section{Scaling behaviour of punctured polygons}

We briefly preview the main results of this section. In subsection 4.1 we study polygons with a finite number of minimal punctures. Our result assumes a certain asymptotic form for the area moment coefficients for unpunctured polygons. This 'assumed' form is known to be true for staircase polygons and many other models and universally accepted as true for self-avoiding polygons. Given this assumption, we prove that the asymptotic behaviour of the area moment coefficients for minimally punctured polygons can be expressed in terms of the asymptotic behaviour of unpunctured polygons. In particular we derive expressions for the leading amplitude of the area moments for punctured polygons in terms of the amplitudes for unpunctured polygons. For staircase polygons this leads to exact formulas for the amplitudes. For self-avoiding polygons the formulas contain certain constants which aren't known exactly but can be estimated numerically to a very high degree of accuracy. In subsection 4.2 we extend the study and proofs to polygons with a finite number of punctures of bounded size and then in subsection 4.3 to models with punctures of arbitrary or unbounded size. Finally in subsection 4.4 we consider the consequences of our results for the area limit laws of punctured polygons and we present conjectures for the scaling functions.

\subsection{Polygons with $r$ minimal punctures}

For polygon models with rational perimeter generating functions, corresponding models with minimal punctures have been studied in [37]. In particular, a method to derive explicit expressions for generating functions of exactly solvable models with a minimal puncture was given [37, Appendix]. It has been applied to Ferrers diagrams, whose perimeter and area generating function satisfies a linear $q$-difference equation, see [37, Eq. (54)]. The method can also be applied to the model of staircase polygons, whose half-perimeter and area generating function $\mathcal{P}(x, q)$ satisfies the quadratic $q$-difference equation

$$
\mathcal{P}(x, q)=\frac{x^{2} q}{1-2 q x-\mathcal{P}(q x, q)} .
$$

Let $\mathcal{P}^{\square(r)}(x, q)$ denote the half-perimeter and area generating function of staircase polygons with $r$ minimal punctures. We have the following result for the case $r=1$.

Fact 1. The half-perimeter and area generating function of staircase polygons with a single minimal puncture $\mathcal{P}^{\square(1)}(x, q)$ is given by

$$
\mathcal{P}^{\square(1)}(x, q)=\frac{x^{4}}{(1-2 q x-\mathcal{P}(q x, q))^{2}}\left(\mathcal{P}(q x, q)-q x \frac{\partial \mathcal{P}}{\partial x}(q x, q)+q \frac{\partial \mathcal{P}}{\partial q}(q x, q)\right),
$$


where $\mathcal{P}(x, q)$ satisfies Eq. (10).

Remarks. i) For a proof of Fact 1, proceed along the lines of [37, Appendix]. We do not give the details, since we are mainly interested in asymptotic results, for which we will give an elementary combinatorial derivation, valid for arbitrary $r$. See Proposition 2 and its subsequent extensions.

ii) For polygons with $r$ punctures, their $k^{\text {th }}$ area moment generating functions are defined by $\mathcal{P}_{k}^{\square(r)}(x)=\left.\left(q \frac{\partial}{\partial q}\right)^{k} \mathcal{P}^{\square(r)}(x, q)\right|_{q=1}$. The above equations can be used to obtain explicit expressions for the area moment generating functions $\mathcal{P}_{k}^{\square(1)}(x)$ by implicit differentiation. The functions $\mathcal{P}_{k}^{\square(1)}(x)$ also appear in section 6.1 .

iii) Assuming that $\mathcal{P}^{\square(1)}(x, q)$ has scaling behaviour of the form

$$
\mathcal{P}^{\square(1)}(x, q) \sim(1-q)^{\theta_{1}} \mathcal{F}^{\square(1)}\left(\left(x_{c}-x\right)(1-q)^{-\phi_{1}}\right)
$$

about $(x, q)=\left(x_{c}, 1\right)$, and the necessary analyticity conditions for the validity of the following calculation, we can express the scaling function $\mathcal{F}^{\square(1)}(s)$ of staircase polygons with a single minimal puncture in terms of the known scaling function $\mathcal{F}(s)$ of staircase polygons Eq. (8). From Eq. (11) we infer that $\theta_{1}=-2 / 3, \phi_{1}=2 / 3$ and

$$
\mathcal{F}^{\square(1)}(s)=\frac{1}{24} s \mathcal{F}^{\prime}(s)-\frac{1}{48} \mathcal{F}(s) .
$$

In principle, the method of [37, Appendix] can be used to analyse the case of several minimal punctures. However, the analysis becomes quite cumbersome. On the other hand, the previous result suggests simple expressions for the scaling functions of models with several punctures in terms of that without a puncture. Moreover, we expect such a phenomenon also to occur for models where an exact solution does not exist or is not known. This is discussed next. We will asymptotically analyse the area moments of a polygon model with punctures and draw conclusions about their possible scaling behaviour.

For a class of punctured self-avoiding polygons, consider their area moment coefficients

$$
p_{m}^{\square(r, k)}:=\sum_{n} n^{k} p_{m, n}^{\square(r)},
$$

where $p_{m, n}^{\square(r)}$ denotes the number of polygons in the class with $r$ minimal punctures, $r \in \mathbb{N}_{0}$, of half-perimeter $m$ and area $n$. For simplicity of notation, we write $p_{m, n}:=p_{m, n}^{\square(0)}$ and $p_{m}^{(k)}:=p_{m}^{\square(0, k)}$. The area moments in the uniform fixed perimeter ensemble are expressed in terms of the area moment coefficients via

$$
\mathbb{E}\left[\left(\widetilde{X}_{m}^{\square(r)}\right)^{k}\right]=\frac{\sum_{n} n^{k} p_{m, n}^{\square(r)}}{\sum_{n} p_{m, n}^{\square(r)}}=\frac{p_{m}^{\square(r, k)}}{p_{m}^{\square(r, 0)}} .
$$


Proposition 2. Assume that, for a class of self-avoiding polygons without punctures, the area moment coefficients $p_{m}^{(k)}$ have the asymptotic form, for $k \in \mathbb{N}_{0}$,

$$
p_{m}^{(k)} \sim A_{k} x_{c}^{-m} m^{\gamma_{k}-1} \quad(m \rightarrow \infty)
$$

for numbers $A_{k}>0, x_{c}>0$ and exponents $\gamma_{k}=(k-\theta) / \phi$, where $\theta$ and $\phi$ are real constants and $0<\phi<1$. Then, the area moment coefficient $p_{m}^{\square(r, k)}$ of the polygon class with $r \geq 1$ minimal punctures is asymptotically given by, for $k \in \mathbb{N}_{0}$,

$$
p_{m}^{\square(r, k)} \sim A_{k}^{(r)} x_{c}^{-m} m^{\gamma_{k}^{(r)}-1} \quad(m \rightarrow \infty),
$$

where $A_{k}^{(r)}=A_{k+r} x_{c}^{2 r} / r$ ! and $\gamma_{k}^{(r)}=\gamma_{k+r}$.

Proof. We will derive upper and lower bounds on $p_{m}^{\square(r, k)}$, which will be shown to coincide asymptotically. Let us call two polygons interacting if their boundary curves have nonempty intersection. An upper bound is obtained by allowing for interaction between all constituents of a punctured polygon. Let a polygon $\mathscr{P}$ of half-perimeter $m-2 r$ and area $n+r$ be given. The number of ways of placing $r$ squares inside $\mathscr{P}$ is clearly less than $(n+r)^{r} / r$ !. We thus have

$$
p_{m}^{\square(r, k)} \leq \widetilde{p}_{m}^{(r, k)}:=\frac{1}{r !} \sum_{n \geq 1} n^{k}(n+r)^{r} p_{m-2 r, n+r}=\frac{1}{r !} \sum_{n \geq r+1}(n-r)^{k} n^{r} p_{m-2 r, n} .
$$

By Bernoulli's inequality, we get for $\widetilde{p}_{m}^{(r, k)}$ the bound

$$
\frac{1}{r !} \sum_{n \geq r+1}\left(n^{k+r}-k r n^{k+r-1}\right) p_{m-2 r, n} \leq \widetilde{p}_{m}^{(r, k)} \leq \frac{1}{r !} \sum_{n \geq r+1} n^{k+r} p_{m-2 r, n} .
$$

For every polygon of perimeter $2 s$ and area $t$ we have $t \geq s-1$. Thus, for $m$ sufficiently large, we can replace the lower bound of summation $r+1$ by zero. In particular, the latter relation is for $m \geq 3 r+2$ equivalent to

$$
\frac{1}{r !}\left(p_{m-2 r}^{(k+r)}-k r p_{m-2 r}^{(k+r-1)}\right) \leq \widetilde{p}_{m}^{(r, k)} \leq \frac{1}{r !} p_{m-2 r}^{(k+r)}
$$

The assumption Eq. (14) on the asymptotic behaviour of $p_{m}^{(k)}$ then implies that

$$
\widetilde{p}_{m}^{(r, k)} \sim \frac{x_{c}^{2 r}}{r !} p_{m}^{(k+r)} \quad(m \rightarrow \infty) .
$$

We derive a lower bound by subtracting from the upper bound an upper bound on the number of square-square and square-boundary interactions. Clearly, square-square interactions are only present for $r>1$. For a given polygon $\mathscr{P}$, the number of square-square interactions of $r$ squares is smaller than the number of interactions between two squares, where the remaining $r-2$ squares may occur at arbitrary positions within the polygon. There are five possible configurations for an interaction between two squares, yielding 
the upper bound $5(n+r)(n+r)^{r-2}$. Thus, the contribution to $\widetilde{p}_{m}^{(r, k)}$ from square-square interactions is bounded from above by

$$
\sum_{n \geq 1} n^{k} 5(n+r)(n+r)^{r-2} p_{m-2 r, n+r}=5(r-1) ! \widetilde{p}_{m}^{(r-1, k)}
$$

which is asymptotically negligible compared to $\widetilde{p}_{m}^{(r, k)}$. Similarly, the number of configurations arising from square-boundary interactions is bounded from above by $\sum_{j=1}^{r} 4^{j}(m-$ $2 r)^{j}(n+r)^{r-j}$. This bound is obtained by estimating the number of configurations of $j$ squares at the boundary by $4^{j}(m-2 r)^{j}$, the factor 4 arising from edge and vertex interactions, the factor $(n+r)^{r-j}$ accounting for arbitrary positions of the remaining $(r-j)$ squares. We thus get an upper bound

$$
\begin{aligned}
\sum_{j=1}^{r} 4^{j}(m-2 r)^{j}(r-j) ! \widetilde{p}_{m}^{(r-j, k)} & \sim \sum_{j=1}^{r} 4^{j} x_{c}^{2 r-2 j}(m-2 r)^{j} p_{m}^{(k+r-j)} \\
& \sim 4 x_{c}^{2 r-2} m p_{m}^{(k+r-1)} \quad(m \rightarrow \infty) .
\end{aligned}
$$

By assumption, the latter bound is asymptotically negligible compared to $\widetilde{p}_{m}^{(r, k)}$. Thus, the lower bound is asymptotically equal to the upper bound, which yields the assertion of the proposition.

Remarks. i) Proposition 2 expresses the asymptotic behaviour of the area moment coefficients of minimally punctured polygons in terms of those of polygons without punctures. The assumption Eq. (14) on the growth of the area moment coefficients of the model without punctures is satisfied for the usual polygon models [35]. The asymptotic behaviour of some models satisfying $\phi=1$, to which Proposition 2 does not apply, has been studied in $[37]$.

ii) As discussed in the previous subsection, the amplitudes $A_{k}$ are related to the amplitudes $f_{k}$ of Eq. (6) by Eq. (5), if $\gamma_{k} \in(-1, \infty) \backslash\{0\}$. For staircase polygons, where $\theta=1 / 3$ and $\phi=2 / 3$, we have explicit expressions for the amplitudes $A_{k}$. More generally, it has been shown $[13,33,34]$ that, for classes of polygon models whose generating function satisfies a $q$-functional equation with a square root as the dominant singularity of their perimeter generating function, we have $f_{k}=c_{k} f_{1}^{k} f_{0}^{1-k}$, where the numbers $c_{k}$ are, for $k \geq 1$, given by

$$
\gamma_{k-1} c_{k-1}+\frac{1}{4} \sum_{l=0}^{k} c_{k-l} c_{l}=0, \quad c_{0}=1 .
$$

The critical point $x_{c}$ as well as $f_{0}$ and $f_{1}$ are model dependent constants. For staircase polygons we have $x_{c}=1 / 4, f_{0}=-1$ and $f_{1}=-1 / 64$.

iii) Rooted self-avoiding polygons are conjectured to also have the exponents $\theta=1 / 3$ and $\phi=2 / 3$. In this case the asymptotic form Eq. (14) and the form of the amplitudes $A_{k}$, given in Eqs. (5) and (16), has been tested for $k \leq 10$ and shown to hold for to a high degree of numerical accuracy [14]. Here $x_{c}=0.14368062927(2)$ is the radius of convergence of the (rooted) SAP half-perimeter generating function, $f_{0}=-0.929607(1)$ and $f_{1}=-x_{c} /(8 \pi)$ 
are the rooted SAP critical amplitudes as in Eq. (6). We conjecture that the asymptotic form (14) holds for rooted SAPs for all values of $k$. Accepting this conjecture to be true, Proposition 2 gives the asymptotic behaviour for rooted self-avoiding polygons with $r$ minimal punctures. By definition, unrooted SAPs have the same amplitudes $A_{k}$.

iv) The crude combinatorial estimates of interactions in the proof of Proposition 2 cannot be used to obtain corrections to the asymptotic behaviour. See also the discussion in the conclusion.

\subsection{Polygons with $r$ punctures of bounded size}

The arguments in the above proof can be applied to obtain results for polygon models with a finite number of punctures of bounded size. The following theorem generalises Proposition 2 and serves as preparation for the next section, where the case of a finite number of punctures of arbitrary size is discussed. For a class of punctured self-avoiding polygons, consider their area moment coefficients

$$
p_{m}^{(r, k, s)}:=\sum_{n} n^{k} p_{m, n}^{(r, s)}
$$

where $p_{m, n}^{(r, s)}$ denotes the number of polygons in the class of half-perimeter $m$ and area $n$ with $r$ punctures, $r \in \mathbb{N}_{0}$, obeying the condition that the sum of the half-perimeter values of the puncturing polygons equals $s$. For simplicity of notation, we write $p_{m, n}:=p_{m, n}^{(0,0)}$, $p_{m}^{(k)}:=p_{m}^{(0, k, 0)}$ and $p_{m}:=p_{m}^{(0)}$.

Theorem 1. Assume that, for a class of self-avoiding polygons without punctures, the area moment coefficients $p_{m}^{(k)}$ have the asymptotic form, for $k \in \mathbb{N}_{0}$,

$$
p_{m}^{(k)} \sim A_{k} x_{c}^{-m} m^{\gamma_{k}-1} \quad(m \rightarrow \infty),
$$

for numbers $A_{k}>0, x_{c}>0$ and $\gamma_{k}=(k-\theta) / \phi$, where $\theta$ and $\phi$ are real constants and $0<\phi<1$. Denote its half-perimeter generating function by $\mathcal{P}(x)=\left(\sum_{m \geq 0} x^{m} p_{m}\right)$. Fix $r \geq 1$ und $s \in \mathbb{N}$ such that $\left[x^{s}\right](\mathcal{P}(x))^{r} \neq 0$. Then, the area moment coefficient $p_{m}^{(r, k, s)}$ of the polygon class with $r$ punctures whose half-perimeter sum equals is asymptotically given by, for $k \in \mathbb{N}_{0}$,

$$
p_{m}^{(r, k, s)} \sim A_{k}^{(r, s)} x_{c}^{-m} m^{\gamma_{k}^{(r)}-1} \quad(m \rightarrow \infty),
$$

where $\gamma_{k}^{(r)}=\gamma_{k+r}$ and $A_{k}^{(r, s)}=\frac{A_{k+r}}{r !} x_{c}^{s}\left[x^{s}\right](\mathcal{P}(x))^{r}$.

Remarks. i) With $s=2$, Theorem 1 reduces to Proposition 2. By summation, we also obtain the asymptotic behaviour for models with $r$ punctures of total half-perimeter less or equal to $s$. Note that we have the formal identity

$$
\sum_{s=0}^{\infty} x^{s}\left[x^{s}\right](\mathcal{P}(x))^{r}=(\mathcal{P}(x))^{r} .
$$


The above expressions are convergent for $|x|<x_{c}$. If $\theta>0$, the sum is also convergent in the limit $x \nearrow x_{c}$.

ii) The remarks following the proof of Proposition 2 also apply to Theorem 1.

Proof. This proof is a direct extension of the proof of Proposition 2 to the case of a finite number of punctures of bounded size. We consider a model of punctured polygons where, for fixed $s$, the $r$ punctures of half-perimeter $s_{i}$ and area $t_{i}$ satisfy $s_{1}+\ldots+s_{r}=s$. We give an asymptotic estimate for $p_{m}^{(r, k, s)}$. Let a polygon $\mathscr{P}$ of half-perimeter $m-|s|$ and of area $n+|\boldsymbol{t}|$, where $|\boldsymbol{s}|=s_{1}+\ldots+s_{r}$ and $|\boldsymbol{t}|=t_{1}+\ldots+t_{r}$, be given. To obtain an upper bound for $p_{m}^{(r, k, s)}$, ignore all interactions between components of a punctured polygon. Recall that two polygons interact if their boundary curves have non-empty intersection. The number of ways of placing $r$ punctures inside $\mathscr{P}$ is clearly smaller than

$$
(n+|\boldsymbol{t}|)^{r} / r !
$$

This bound is obtained by considering the number of ways of placing the lower left corner of each puncture on each square plaquette inside the polygon. Note that, unlike in the proof of Proposition 2, this bound also counts configurations where punctures protrude from the boundary of $\mathscr{P}$. We will compensate for these over-counted configurations when deriving a lower bound for $p_{m}^{(r, k, s)}$. We have

$$
\begin{aligned}
& p_{m}^{(r, k, s)} \leq \widetilde{p}_{m}^{(r, k, s)}:=\frac{1}{r !} \sum_{|\boldsymbol{s}|=s} \sum_{t_{i}} \sum_{n \geq 1} n^{k}(n+|\boldsymbol{t}|)^{r} p_{m-|\boldsymbol{s}|, n+|\boldsymbol{t}|} \prod_{i=1}^{r} p_{s_{i}, t_{i}} \\
& =\frac{1}{r !} \sum_{|\boldsymbol{s}|=s} \sum_{t_{i}} \sum_{n \geq|\boldsymbol{t}|+1}(n-|\boldsymbol{t}|)^{k} n^{r} p_{m-|\boldsymbol{s}|, n} \prod_{i=1}^{r} p_{s_{i}, t_{i}},
\end{aligned}
$$

where the first sum is over the variables $s_{1}, \ldots, s_{r}$ subject to the restriction $|\boldsymbol{s}|=s$, and the second sum is over all values of the variables $t_{1}, \ldots, t_{r}$. Note that, for $m$ fixed, all sums are finite. Invoking Bernoulli's inequality, we obtain the bound

$$
\begin{gathered}
\frac{1}{r !} \sum_{|\boldsymbol{s}|=s} \sum_{t_{i}} \sum_{n \geq|\boldsymbol{t}|+1}\left(n^{k+r}-k|\boldsymbol{t}| n^{k+r-1}\right) p_{m-|\boldsymbol{s}|, n} \prod_{i=1}^{r} p_{s_{i}, t_{i}} \leq \widetilde{p}_{m}^{(r, k, s)} \\
\leq \frac{1}{r !} \sum_{|\boldsymbol{s}|=s} \sum_{t_{i}} \sum_{n \geq|\boldsymbol{t}|+1} n^{k+r} p_{m-|\boldsymbol{s}|, n} \prod_{i=1}^{r} p_{s_{i}, t_{i}} .
\end{gathered}
$$

Consider first the asymptotic behaviour of the expression

$$
\widetilde{a}_{m, s}:=\sum_{|\boldsymbol{s}|=s} \sum_{t_{i}} \sum_{n \geq|\boldsymbol{t}|+1} n^{k+r} p_{m-|\boldsymbol{s}|, n} \prod_{i=1}^{r} p_{s_{i}, t_{i}} .
$$

If $m \geq|\boldsymbol{s}|^{2}+|\boldsymbol{s}|+2$, then the lower bound of summation on the index $n$ may be replaced by zero. This follows from the estimate $t_{i} \leq s_{i}^{2}$, being valid for every self-avoiding polygon of half-perimeter $s_{i}$ and area $t_{i}$. Thus $|\boldsymbol{t}| \leq|\boldsymbol{s}|^{2}$, and we argue that $n \geq m-|\boldsymbol{s}|-1 \geq$ $|\boldsymbol{s}|^{2}+1 \geq|\boldsymbol{t}|+1$. We thus get for $m$ sufficiently large

$$
\widetilde{a}_{m, s}=\sum_{|\boldsymbol{s}|=s} p_{m-|\boldsymbol{s}|}^{(k+r)} \prod_{i=1}^{r} p_{s_{i}} \sim p_{m}^{(k+r)}\left(\sum_{|\boldsymbol{s}|=s} x_{c}^{|\boldsymbol{s}|} \prod_{i=1}^{r} p_{s_{i}}\right) \quad(m \rightarrow \infty),
$$


where the sum in brackets is finite. We now analyse the second term in the estimate derived from the Bernoulli inequality. To this end, define

$$
\widetilde{b}_{m, s}:=\sum_{|\boldsymbol{s}|=s} \sum_{t_{i}} \sum_{n \geq|\boldsymbol{t}|+1}|\boldsymbol{t}| n^{k+r-1} p_{m-|\boldsymbol{s}|, n} \prod_{i=1}^{r} p_{s_{i}, t_{i}} .
$$

Using the estimate $|\boldsymbol{t}| \leq|\boldsymbol{s}|^{2}$, we get

$$
\widetilde{b}_{m, s} \leq s^{2}\left(\sum_{|\boldsymbol{s}|=s} p_{m-|\boldsymbol{s}|}^{(k+r-1)} \prod_{i=1}^{r} p_{s_{i}}\right) \sim s^{2} p_{m}^{(k+r-1)}\left(\sum_{|\boldsymbol{s}|=s} x_{c}^{|s|} \prod_{i=1}^{r} p_{s_{i}}\right) \quad(m \rightarrow \infty) .
$$

Now set $b_{m, s}:=\widetilde{b}_{m, s} /\left(x_{c}^{-m} m^{\gamma_{k}^{(r)}-1}\right)$. The above estimate yields $\lim _{m \rightarrow \infty} b_{m, s}=0$, since $0<\phi<1$.

We now derive a lower bound for $p_{m}^{(r, k, s)}$ by subtracting from $\widetilde{p}_{m}^{(r, k, s)}$ an upper bound on the contributions arising from puncture-puncture interactions and from punctureboundary interactions. We will show that the lower bound coincides asymptotically with the upper bound, which then implies the assertion of the theorem

$$
p_{m}^{(k, r, s)} \sim \frac{A_{k+r}}{r !}\left(\sum_{|\boldsymbol{s}|=s} x_{c}^{|\boldsymbol{s}|} \prod_{i=1}^{r} p_{s_{i}}\right) x_{c}^{-m} m^{\gamma_{k+r}-1} \quad(m \rightarrow \infty) .
$$

For any polygon $\mathscr{P}$, the number of puncture-puncture interactions between $r>1$ punctures is smaller than the number of puncture-puncture interactions of two punctures with the remaining $r-2$ punctures occuring at arbitrary positions in the polygon. We thus get the upper bound

$$
\left(t_{1}+4 s_{1}\right) t_{2}(n+|\boldsymbol{t}|)(n+|\boldsymbol{t}|)^{r-2} \leq 6 t_{1} t_{2}(n+|\boldsymbol{t}|)^{r-1},
$$

where we used $t_{1} \geq s_{1}-1$. The factor $\left(t_{1}+4 s_{1}\right) t_{2}$ bounds the number of configurations of two interacting punctures, and the factor $(n+|\boldsymbol{t}|)^{r-2}$ arises from allowing arbitrary positions of the remaining $r-2$ punctures. Define

$$
\widetilde{c}_{m, s}:=\sum_{|\boldsymbol{s}|=s} \sum_{t_{i}} \sum_{n} t_{1} t_{2} n^{k}(n+|\boldsymbol{t}|)^{r-1} p_{m-|\boldsymbol{s}|, n+|\boldsymbol{t}|} \prod_{i=1}^{r} p_{s_{i}, t_{i}} \leq s^{4}\left(\sum_{|\boldsymbol{s}|=s} p_{m-|\boldsymbol{s}|}^{(k+r-1)} \prod_{i=1}^{r} p_{s_{i}}\right),
$$

where we used $t_{i} \leq|\boldsymbol{t}| \leq|\boldsymbol{s}|^{2}$ for the last inequality. Setting $c_{m, s}:=\widetilde{c}_{m, s} /\left(x_{c}^{-m} m^{\gamma_{k}^{(r)}-1}\right)$, we infer that $\lim _{m \rightarrow \infty} c_{m, s}=0$. We have shown that for fixed $s$ the puncture-puncture interactions are asymptotically irrelevant.

We finally estimate the puncture-boundary interactions. This is done similarly to the above treatment of puncture-puncture interactions. The number of puncture-boundary interactions is bounded from above by

$$
\sum_{j=1}^{r} 4^{j}(m-|s|)^{j} s_{1} \cdot \ldots \cdot s_{j}(n+|\boldsymbol{t}|)^{r-j}
$$


where $j$ punctures interact with the boundary, each contributing a factor $4(m-|s|) s_{i}$, and $r-j$ punctures have arbitrary positions, each contributing a factor $(n+|\boldsymbol{t}|)$. Note that the over-counted configurations in $\widetilde{p}_{m}^{(r, k, s)}$, which protrude from the boundary, are compensated for by the above estimate. Define

$$
\begin{gathered}
\widetilde{d}_{m, s}:=\sum_{|\boldsymbol{s}|=s} \sum_{t_{i}} \sum_{n}(m-|\boldsymbol{s}|)^{j} n^{k}(n+|\boldsymbol{t}|)^{r-j} s_{1} \cdot \ldots \cdot s_{j} p_{m-|\boldsymbol{s}|, n+|\boldsymbol{t}|} \prod_{i=1}^{r} p_{s_{i}, t_{i}} \\
\leq(m-s)^{j} s^{j}\left(\sum_{|\boldsymbol{s}|=s} p_{m-|\boldsymbol{s}|}^{(k+r-j)} \prod_{i=1}^{r} p_{s_{i}}\right) .
\end{gathered}
$$

Defining $d_{m, s}:=\widetilde{d}_{m, s} /\left(x_{c}^{-m} m_{k}^{\gamma_{k}^{(r)}-1}\right)$, we infer that $\lim _{m \rightarrow \infty} d_{m, s}=0$. We have shown that for fixed $s$ the puncture-boundary interactions are asymptotically irrelevant. This completes the proof.

\subsection{Polygons with $r$ punctures of arbitrary size}

For a class of punctured self-avoiding polygons, consider for $k \in \mathbb{N}_{0}$ their area moment coefficients

$$
p_{m}^{(r, k)}:=\sum_{n} n^{k} p_{m, n}^{(r)}
$$

where $p_{m, n}^{(r)}:=\sum_{s=0}^{\infty} p_{m, n}^{(r, s)}<\infty$ denotes the number of polygons in the class of halfperimeter $m$ and area $n$ with $r$ punctures of arbitrary size, $r \in \mathbb{N}_{0}$. For simplicity of notation, we write $p_{m, n}=p_{m, n}^{(0)}$ and $p_{m}^{(k)}=p_{m}^{(0, k)}$. In the sequel, we will use the area moment generating functions $\mathcal{P}_{k}(x)=\sum p_{m}^{(k)} x^{m}$ of the model without punctures.

Theorem 2. Assume that, for a class of self-avoiding polygons without punctures, the area moment coefficients $p_{m}^{(k)}$ have the asymptotic form, for $k \in \mathbb{N}_{0}$,

$$
p_{m}^{(k)} \sim A_{k} x_{c}^{-m} m^{\gamma_{k}-1} \quad(m \rightarrow \infty)
$$

for numbers $A_{k}>0, x_{c}>0$ and $\gamma_{k}=(k-\theta) / \phi$, where $0<\phi<1$. Let $\mathcal{P}_{k}(x)=\sum p_{m}^{(k)} x^{m}$ denote the $k^{\text {th }}$ area moment generating function.

Then, the area moment coefficient $p_{m}^{(r, k)}$ of the polygon class with $r \geq 1$ punctures of arbitrary size is, for $k \in \mathbb{N}_{0}$, bounded from above by

$$
p_{m}^{(r, k)} \leq \frac{\left[x^{m}\right] \mathcal{P}_{k+r}(x)\left(\mathcal{P}_{0}(x)\right)^{r}}{r !}
$$

For finite critical perimeter generating functions, characterised by $\theta>0, p_{m}^{(r, k)}$ is asymptotically given by, for $k \in \mathbb{N}_{0}$,

$$
p_{m}^{(r, k)} \sim \frac{\left[x^{m}\right] \mathcal{P}_{k+r}(x)\left(\mathcal{P}_{0}(x)\right)^{r}}{r !} \sim A_{k}^{(r)} x_{c}^{-m} m^{\gamma_{k+r}-1} \quad(m \rightarrow \infty),
$$

where the amplitudes $A_{k}^{(r)}$ are given by

$$
A_{k}^{(r)}=\frac{A_{k+r}\left(\mathcal{P}_{0}\left(x_{c}\right)\right)^{r}}{r !}
$$


where $\mathcal{P}_{0}\left(x_{c}\right):=\lim _{x / x_{c}} \mathcal{P}_{0}(x)<\infty$ is the critical amplitude of the half-perimeter generating function.

Remarks. i) The asymptotic form Eq. (19) is formally obtained from Theorem 1 in the limit of infinite puncture size, see Remark i) after Theorem 1. This observation is also the main ingredient of the following proof, by noting that the upper bound has the same asymptotic behaviour.

ii) For staircase polygons, where $\theta=1 / 3$ and $\phi=2 / 3$, the assumptions of Theorem 2 are satisfied. For self-avoiding polygons, we have the numerically very well established values $\theta=1$ and $\phi=2 / 3$, which we believe to describe the asymptotic behaviour of SAPs. For models satisfying $\theta<0$, the upper bound generally does not coincide asymptotically with $p_{m}^{(r, k)}$. An example of failure is rectangles with a single puncture.

Proof. We obtain as in the proof of Theorem 1 an upper bound $\widetilde{p}_{m}^{(r, k)}$ for the area moment coefficients $p_{m}^{(r, k)}$. It is given by

$$
p_{m}^{(r, k)} \leq \widetilde{p}_{m}^{(r, k)}:=\frac{1}{r !} \sum_{s=0}^{m} \sum_{|\mathbf{s}|=s} p_{m-|\mathbf{s}|}^{(k+r)} \prod_{i=1}^{r} p_{s_{i}}=\frac{1}{r !}\left[x^{m}\right] \mathcal{P}_{k+r}(x)\left(\mathcal{P}_{0}(x)\right)^{r} .
$$

Assume in the following that $\theta>0$. The asymptotic behaviour of the rhs of (19) follows by $r$-fold application of Lemma 1, which is given in the appendix. Note that, for $M$ arbitrary, we have by definition

$$
p_{m}^{(r, k)} \geq \sum_{s=0}^{M} p_{m}^{(r, k, s)}
$$

where $p_{m}^{(r, k, s)}$ is the number of $r$-punctured polygons, whose punctures have total perimeter equal to $s$. Theorem 1 implies that the above sum is, for $M$ sufficiently large, asymptotically in $m$, arbitrarily close to the upper bound $\widetilde{p}_{m}^{(r, k)}$. See also the remark following Theorem 1. This yields the statement of the theorem.

\subsection{Limit distribution of area and scaling function conjectures}

We first discuss the implications of the previous results on the asymptotic area law of polygon models with punctures. By an application of Proposition 1, Theorem 1 and Theorem 2 immediately yield the following result:

Theorem 3. Assume that, for a class of self-avoiding polygons without punctures, the area moment coefficients $p_{m}^{(k)}$ have the asymptotic form, for $k \in \mathbb{N}_{0}$,

$$
p_{m}^{(k)} \sim A_{k} x_{c}^{-m} m^{\gamma_{k}-1} \quad(m \rightarrow \infty)
$$

for numbers $A_{k}>0, x_{c}>0$ and $\gamma_{k}=(k-\theta) / \phi$, where $0<\phi<1$. Assume further that the numbers $A_{k}$ satisfy the Carleman condition

$$
\sum_{k \geq 0}\left(A_{2 k}\right)^{-1 / 2 k}=+\infty
$$

Denote the half-perimeter generating function of the model by $\mathcal{P}(x)=\left(\sum_{m \geq 0} x^{m} p_{m}\right)$. 
i) Consider for $r \geq 1$ the corresponding model with $r$ punctures of bounded size, whose half-perimeter sum equals $s \in \mathbb{N}$, such that $\left[x^{s}\right](\mathcal{P}(x))^{r} \neq 0$. Denote the random variables of area in the uniform fixed perimeter ensemble by $\widetilde{X}_{m}^{(r, s)}$. Then, we have convergence in distribution,

$$
\frac{\tilde{X}_{m}^{(r, s)}}{m^{1 / \phi}} \stackrel{d}{\longrightarrow} X^{(r, s)} \quad(m \rightarrow \infty)
$$

for a uniquely defined random variable $X^{(r, s)}$ with moments

$$
\mathbb{E}\left[\left(X^{(r, s)}\right)^{k}\right]=\frac{A_{k}^{(r, s)}}{A_{r}},
$$

where the numbers $A_{k}^{(r, s)}$ are those of Theorem 1. We also have moment convergence.

ii) Let $\widetilde{X}_{m}^{(r)}$ denote the random variable of area in the fixed perimeter ensemble for the model with $r \geq 1$ punctures of unbounded size. If $\theta>0$, then we have convergence in distribution,

$$
\frac{\tilde{X}_{m}^{(r)}}{m^{1 / \phi}} \stackrel{d}{\longrightarrow} X^{(r)} \quad(m \rightarrow \infty)
$$

for a uniquely defined random variable $X^{(r)}$ with moments

$$
\mathbb{E}\left[\left(X^{(r)}\right)^{k}\right]=\frac{A_{k}^{(r)}}{A_{r}},
$$

where the numbers $A_{k}^{(r)}$ are those of Theorem 2. We also have moment convergence.

iii) If $\theta>0$, the random variables $X^{(r)}$ and $X^{(r, s)}$ are related by

$$
x_{c}^{s}\left[x^{s}\right](\mathcal{P}(x))^{r} X^{(r)}=\left(\mathcal{P}\left(x_{c}\right)\right)^{r} X^{(r, s)},
$$

where $\mathcal{P}(x)$ is the half-perimeter generating function of the polygon model without punctures, and where $\mathcal{P}\left(x_{c}\right)=\lim _{x / x_{c}} \mathcal{P}(x)<\infty$.

Remarks. i) For a given polygon model satisfying the assumptions of Theorem 3, the area moments satisfy asymptotically

$$
\frac{\mathbb{E}\left[\left(\widetilde{X}_{m}^{(r)}\right)^{k}\right]}{k !} \sim D_{k}^{(r)} m^{k / \phi} \quad(m \rightarrow \infty),
$$

for positive numbers $D_{k}^{(r)}$. For classes of polygon models whose generating function satisfies a $q$-functional equation with a square root as the dominant singularity of their perimeter generating function, the amplitude ratios $D_{k}^{(r)} /\left[D_{1}^{(r)}\right]^{k}$ are universal, i.e., independent 


\begin{tabular}{cccc}
\hline \hline Amplitude & $r=0$ & $r=1$ & $r=2$ \\
\hline$D_{2} / D_{1}^{2}$ & $0.530518 \times 10^{-0}$ & $0.530143 \times 10^{-0}$ & $0.529356 \times 10^{-0}$ \\
$D_{3} / D_{1}^{3}$ & $0.198944 \times 10^{-0}$ & $0.198369 \times 10^{-0}$ & $0.197361 \times 10^{-0}$ \\
$D_{4} / D_{1}^{4}$ & $0.592379 \times 10^{-1}$ & $0.588127 \times 10^{-1}$ & $0.581533 \times 10^{-1}$ \\
$D_{5} / D_{1}^{5}$ & $0.149079 \times 10^{-1}$ & $0.146994 \times 10^{-1}$ & $0.144042 \times 10^{-1}$ \\
$D_{6} / D_{1}^{6}$ & $0.329453 \times 10^{-2}$ & $0.321705 \times 10^{-2}$ & $0.311511 \times 10^{-2}$ \\
$D_{7} / D_{1}^{7}$ & $0.655743 \times 10^{-3}$ & $0.632288 \times 10^{-3}$ & $0.603260 \times 10^{-3}$ \\
$D_{8} / D_{1}^{8}$ & $0.119654 \times 10^{-3}$ & $0.113600 \times 10^{-3}$ & $0.106501 \times 10^{-3}$ \\
$D_{9} / D_{1}^{9}$ & $0.202754 \times 10^{-4}$ & $0.189015 \times 10^{-4}$ & $0.173673 \times 10^{-4}$ \\
$D_{10} / D_{1}^{10}$ & $0.322150 \times 10^{-5}$ & $0.294132 \times 10^{-5}$ & $0.264251 \times 10^{-5}$ \\
\hline \hline
\end{tabular}

Table 1: Universal amplitude ratios for staircase polygons with $r$ punctures.

of the constants $f_{0}, f_{1}$ and $x_{c}$, which characterise the underlying model $[13,33,34]$. This follows from Eqs. (5) and (16) by a straightforward calculation. The numbers are listed in Table 1 for small values of $r$. Note that the same numbers appear for punctures of bounded size.

ii) For the above class of models, explicit expressions for the asymptotic behaviour of their moment generating functions and their probability distributions can be derived from the area amplitude series via inverse Laplace transform techniques. Since the resulting expressions are quite cumbersome, we do not give them here. For $r=0$ the corresponding limit distribution of area is the Airy distribution [28, 29,30]. The extension to $r \geq 1$ is straightforward. As mentioned above, for $r=0$ the amplitude ratios are found to coincide with those of (rooted) self-avoiding polygons to a high degree of numerical accuracy [14]. If the conjecture holds true that they agree exactly, then the above expressions for limit distributions also appear for rooted self-avoiding polygons, for all values of $r$. See Section 6 for a detailed numerical analysis.

We now discuss the relations between the area amplitude series $F(z)$ of the polygon model without punctures and $F^{(r)}(z)$ of the polygon model with $r$ punctures. Since all of our models have an entire moment generating function, the Carleman condition is satisfied, and Theorem 1 and Theorem 2 yield, by a straightforward calculation, the following result.

Theorem 4. Assume that, for a class of self-avoiding polygons, the polygon model without punctures has an area amplitude series, given by

$$
F(z)=\sum_{k \geq 0} f_{k} z^{-\gamma_{k}}
$$

where $\gamma_{k}=(k-\theta) / \phi \in(-1, \infty) \backslash\{0\}$ and $0<\phi<1$, and where the numbers $f_{k} \neq 0$ are related to the amplitudes $A_{k}$ in Proposition 1 via Eq. (5). Denote the half-perimeter generating function of the model by $\mathcal{P}(x)=\sum_{m \geq 0} x^{m} p_{m}$. 
i) Assume that $r \geq 1$ and $s \in \mathbb{N}$ are given such that $\left[x^{s}\right](\mathcal{P}(x))^{r} \neq 0$. Then, the corresponding model of punctured polygons with $r$ punctures of bounded size $s$ has an area amplitude series, given by

$$
F^{(r, s)}(z)=\sum_{k \geq 0} f_{k}^{(r)} z^{-\gamma_{k}^{(r)}}
$$

where $\gamma_{k}^{(r)}=\left(k-\theta_{r}\right) / \phi_{r}$. We have $\theta_{r}=\theta-r, \phi_{r}=\phi$, and

$$
F^{(r, s)}(z)=\frac{(-1)^{r}}{r !} x_{c}^{s}\left[x^{s}\right](\mathcal{P}(x))^{r} \sum_{k \geq r}(k)_{r} f_{k} z^{-\gamma_{k}}
$$

ii) If $\theta>0$, the corresponding model of punctured polygons with $r \geq 1$ punctures of arbitrary size has an area amplitude series, given by

$$
F^{(r)}(z)=\frac{(-1)^{r}}{r !}\left(\mathcal{P}\left(x_{c}\right)\right)^{r} \sum_{k \geq r}(k)_{r} f_{k} z^{-\gamma_{k}},
$$

where $\mathcal{P}\left(x_{c}\right)=\lim _{x / x_{c}} \mathcal{P}(x)<\infty$.

Remarks. i) Eq. (21) allows one to derive explicit expressions for the area amplitude series in terms of $F(z)$. For models where $\theta=1 / 3$ and $\phi=2 / 3$ such as staircase polygons, the area amplitude series $F(z)$ satisfies the Riccati equation

$$
F(z)^{2}-4 f_{1} F^{\prime}(z)-f_{0}^{2} z=0 .
$$

This can be used to show that $F^{(r, s)}(z)$ (and also $F^{(r)}(z)$ ) is of the form

$$
F^{(r, s)}(z)=\sum_{k=0}^{r+1} p_{k, r}(z) F(z)^{k},
$$

where $p_{k, r}(z)$ are polynomials in $z$ of degree not exceeding $\lceil 3 r / 2\rceil$, and $p_{r+1, r}(z)$ is not identically vanishing. Simple expressions for the polynomials $p_{k, r}(z)$ are not apparent. We note, however, that such expressions appear as correction-to-scaling functions of the underlying polygon models without punctures [13].

ii) The model of rooted self-avoiding polygons has been found numerically to have the same type of area amplitude series as staircase polygons (with different constants $f_{0}$ and $f_{1}$ ). Similar considerations apply to the model of unrooted self-avoiding polygons starting from Eq. (9).

We finally discuss the scaling function conjectures implied by the results of the previous subsections. For staircase polygons, the area amplitude function satisfies the differential equation (22). This differential equation has a unique solution $\mathcal{F}(z)$ analytic for $\Re(z) \geq 0$, 
having $F(z)$ as an asymptotic expansion at infinity, $\mathcal{F}(z) \sim F(z)$ as $z \rightarrow \infty$. The function $\mathcal{F}(z)$ is explicitly given by

$$
\mathcal{F}(z)=-4 f_{1} \frac{\mathrm{d}}{\mathrm{d} z} \log \mathrm{Ai}\left(\left(\frac{f_{0}}{4 f_{1}}\right)^{2 / 3} z\right),
$$

and this function coincides with the scaling function of staircase polygons Eq. (8).

In analogy to the above observation, we conjecture that the area amplitude series for punctured staircase polygons determine their scaling functions. Likewise, we conjecture that the area amplitude series for punctured rooted self-avoiding polygons determine their scaling functions.

Conjecture 1. Let $r \geq 1$ and $s \geq 2$ be given. For staircase polygons and rooted selfavoiding polygons, the area amplitude series $F^{(r, s)}(z)$ and $F^{(r)}(z)$ of Theorem 4 uniquely define functions $\mathcal{F}^{(r, s)}(z)$ and $\mathcal{F}^{(r)}(z)$ analytic for $\Re(z)>z_{0}$ and non-analytic at $z=$ $z_{0}$, for some negative real number $z_{0}<0$. We conjecture that the functions $\mathcal{F}^{(r, s)}(z)$ : $\left(z_{0}, \infty\right) \rightarrow \mathbb{R}$ and $\mathcal{F}^{(r)}(z):\left(z_{0}, \infty\right) \rightarrow \mathbb{R}$ are scaling functions as in Definition 2,

$$
\begin{aligned}
\mathcal{P}^{(r, s)}(x, q) & \sim(1-q)^{1 / 3-r} \mathcal{F}^{(r, s)}\left(\frac{x_{c}-x}{(1-q)^{2 / 3}}\right) \\
\mathcal{P}^{(r)}(x, q) & \sim(1-q)^{1 / 3-r} \mathcal{F}^{(r)}\left(\frac{x_{c}-x}{(1-q)^{2 / 3}}\right) .
\end{aligned}
$$

Remark. The above conjecture has the following implications.

i) Staircase polygons with a single minimal puncture specialise to Eq. (12).

ii) Up to constant factors, the scaling form of the model with $r$ punctures is obtained as the $r^{\text {th }}$ derivative w.r.t. $q$ of the scaling form of the model without punctures, as can be proved by induction. As derivatives can be interpreted combinatorially as marking, this reflects the fact underlying the proofs in this section that punctures may be regarded as being asymptotically independent, and boundary effects do not play a role asymptotically. iii) Ignoring questions of analyticity, a (formal) calculation yields that the functions $\mathcal{F}^{(r)}$ (and $\mathcal{F}^{(r, s)}$ ) lead, for both staircase polygons and (unrooted) self-avoiding polygons, to the same critical exponents in the branched polymer phase as those conjectured previously $[21,16]$. These are obtained from the singular behaviour of $\mathcal{F}^{(r)}$ about the singularity of smallest modulus on the negative axis, i.e., at the first zero of the Airy-function on the negative axis, see [13, Sec 1]. The fact that $\mathcal{P}^{(r)}(x, q)$ is obtained from $\mathcal{P}(x, q)$ by $r$-fold differentiation yields the result.

\section{Computer enumerations}

Here we briefly outline which algorithms were used to derive the series expansions for the area moments of punctured polygons. In most cases (SAPs and punctured staircase polygons) the algorithms are simple generalisations of previous algorithms already described in detail in other papers, referenced below. In these cases we give brief details of 

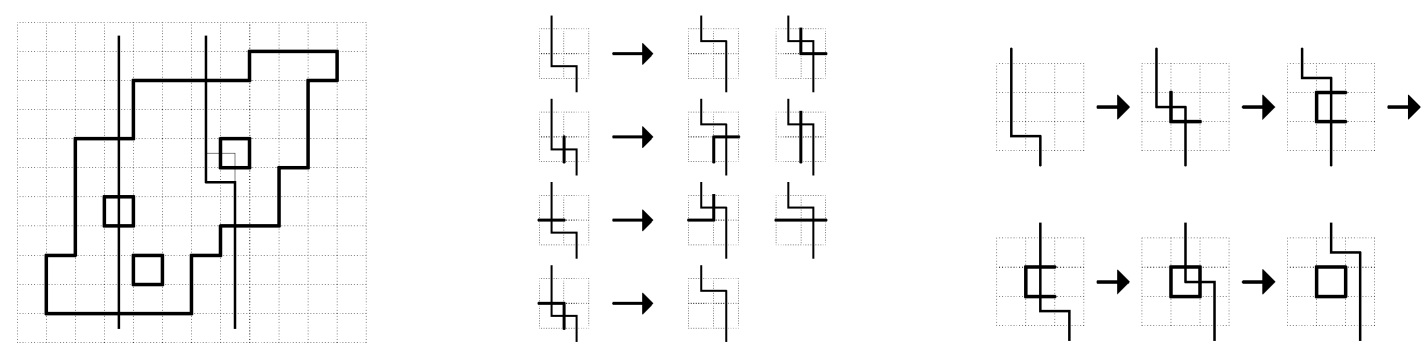

Figure 3: Illustration of the transfer matrix boundary line and local updating rules.

the length of the series and the amount of CPU time used. Only in the case of staircase polygons with minimal punctures did we write a new specific algorithm which we shall describe in some detail.

The series for punctured self-avoiding polygons were calculated using a simple generalisation of the parallel version of the algorithm we used previously to enumerate ordinary SAPs [38]. In each case (SAPs with one or two minimal punctures and SAPs with one or two arbitrary punctures) we calculated the area moments up to $k=10$ for SAPs to total perimeter 100. Since the smallest such SAPs have perimeter 16 and 24 this results in series with 42 and 38 non-zero terms, respectively. The total CPU time required was about 5000 hours for each of the once punctured SAP problems and up to 3000 hours for the twice punctured problems. The bulk of these calculations were performed on the old facility of the Australian Partnership for Advanced Computing (APAC), which was a Compaq Server Cluster with ES45 nodes with $1 \mathrm{GHz}$ Alpha chips (this facility has since been replaced by an SGI Altix cluster).

In [22] we used a very efficient algorithm to enumerate once punctured staircase polygons. The algorithm is easily generalised in order to calculate area moments which we have done to perimeter $718(k=1), 598(k=2)$ and $506(k=3$ to 10$)$. It is also quite straight-forward to generalise the algorithm to count twice punctured staircase polygons and in this case we obtained the series to perimeter 502 for $k=0$, perimeter 450 for $k=1$ and 2 and to perimeter 302 for $k=3$ to 10. It is also easy to extract data for staircase polygons with punctures of fixed combined perimeter. In each case we used around 1000 CPU hours on the APAC Altix cluster which use 1.6GHz Intel Itanium 2 chips.

Finally we describe the algorithm used to enumerate minimally punctured staircase polygons. The algorithm is based on so-called transfer matrix techniques. The basic idea is to count the number of polygons by bisecting the lattice with a boundary line. In the left panel of Fig. 3 we show how such a boundary (the first medium thick line) will intersect the polygon in several places. The first and last occupied edges intersected by the boundary line are the directed walks constituting the outer staircase polygon. The other occupied edges (if any) belong to the minimal punctures. In a calculation to maximal half-perimeter $m$ we need only consider intersections with widths up to $w=m / 2$. Any intersection pattern (or signature) can be specified by a string of occupation variables, $S=\left\{\sigma_{0}, \sigma_{1}, \ldots \sigma_{w}\right\}$, where $\sigma_{i}=0,1$ or 2 if edge number $i$ is empty, an occupied outer 
edge or an occupied edge part of a minimal puncture, respectively. We could use the same symbol for all occupied edges but it is convenient to explicitly distinguish between the two cases. For each signature we keep a generating function which keeps track of the number of configurations (to the left of the boundary line), that is, it counts the number of possible partially completed polygons with a given signature. In order to count the total number of punctured polygons we move the boundary line to the right column by column with each column built up one vertex at a time. In the left panel of Fig. 3 we have also shown a typical move of the boundary line, which starts in the position given by the second medium thick line and where we add two new edges to the lattice by moving the kink in the boundary line to the position given by the thin lines. As we move the boundary line to a new position we calculate the associated generating functions (the updating rules will be given below). Formally we can view this transformation between signatures as a matrix multiplication (hence our use of the nomenclature transfer matrix algorithm). However, as can be readily seen, the transfer matrix is extremely sparse and there is no reason to list it explicitly (it is given implicitly by the updating rules).

We start the calculation with the initial signature $\{1,1,0, \ldots, 0\}$, which corresponds to inserting the two steps of the outer walks in the lower left corner (the count of this configuration is 1). As the boundary line is moved it passes over a vertex and the updating depends on the states of the edges below and to the left of this vertex. After the move we 'insert' the edges to the right and above the vertex. There are four possible local configurations of the 'incoming' edges as illustrated in the middle panels of Fig. 3: Both edges are empty, one of the edges is occupied and the other edge is empty or both edges are occupied.

Both edges empty: If both incoming edges are empty then both outgoing edges can be empty. Else we may insert two new steps which must be part of a minimal puncture (the outgoing edges are in state ' 2 '). This is only possible if the vertex is in the interior of the polygon (there is an edge in state ' 1 ' both below and above the vertex).

Left edge empty, bottom edge occupied: The walk occupying the incoming edge must be continued along an outgoing edge. If the occupied edge is part of the external polygon (in state ' 1 ') there are no restrictions. If the occupied edge is part of a minimal puncture the walk can only be continued along the edge to the right of the vertex (otherwise we would not get a minimal puncture).

Left edge occupied, bottom edge empty: This is similar to the previous case except that an edge in state ' 2 ' must be continued along the edge above the vertex.

Both edges occupied: If both edges are in state ' 2 ' we close the puncture and the new edges are empty. If the incoming edges are in state ' 1 ' we have closed the outer polygon and then we add the count to the running total for the generating function.

In the last panel of Fig. 3 we show how the updating rules given above through a sequence of moves of the boundary line gives rise to a minimal puncture. 
The perimeter of the completed polygon is given by the position of the boundary line when the polygon is closed, e.g., if we have taken $k$ steps in the $x$-direction (completed $k$ columns) and moved the kink $l$ steps in the $y$-direction then the outer half-perimeter is $k+l$ and the total half-perimeter in $k+l+2 r$. So we need only keep track of the number of punctures $r$. This is done by associating a truncated polynomial $P_{S}(x)=\sum p_{r} x^{r}$ with each signature, where the coefficient $p_{r}$ is the number of partially completed polygons with $r$ punctures of the signature $S$. As a new signature $S^{\prime}$ is created from $S$ we set $P_{S^{\prime}}(x)=P_{S^{\prime}}(x)+x^{\delta} P_{S}(x)$, where $\delta=1$ if an additional puncture is inserted (as in the first case described above) or 0 otherwise. The extension to the calculation of area moments is described in [39].

\section{$6 \quad$ Numerical analysis}

We now turn to our numerical analysis of the series for punctured polygons. In section 6.1 we use our series to determine numerically the exact area moment generating functions for minimally punctured staircase polygons with up to 5 punctures and $k \leq 10$. The resulting exact expressions for the leading amplitudes are in complete agreement with the formula derived in Proposition 2. In section 6.2 we extend the study to staircase polygons with one puncture of fixed size and two punctures of fixed combined size. Again we find the exact generating functions and confirm the formula for the amplitude given in Theorem 1. Next, in section 6.3, we analyse area moments for staircase polygons with a single puncture of arbitrary size. Guided by results obtained from an analysis of the conjectured exact ODE [22] satisfied by the perimeter generating function we carry out a careful numerical analysis of the area moment coefficients. This allows to obtain accurate estimates for the leading amplitudes and we confirm the results of Theorem 2 to at least 15 significant digits. Then, in section 6.4 we extend our study to staircase polygons with two staircase punctures of arbitrary size and we again find good agreement with the exact results. Intriguingly, we find in all of the above cases, that the amplitude of the leading correction term is a constant times the corresponding amplitude with one less puncture. Finally, in section 6.5 we present the results of our analysis of self-avoiding polygons with one and two punctures (minimal as well as arbitrary). In this case the numerical evidence is not quite as convincing, but we do find that the numerical estimates agree with the exact formulas to at least 3-4 significant digits.

\subsection{Staircase polygons with minimal punctures}

In [16] it was found that the half-perimeter generating function of staircase polygons with a single minimal puncture is:

$$
\mathcal{P}^{\square(1)}(x)=\frac{1-8 x+20 x^{2}-16 x^{3}+2 x^{4}}{2(1-4 x)}-\frac{1-6 x+10 x^{2}-4 x^{3}}{2 \sqrt{1-4 x}} .
$$

This result is also derivable from Eq. (11), which gives a functional equation for the areaperimeter generating function. It is thus plausible to expect that the generating function 
of staircase polygons with $r$ minimal punctures is of a similar form

$$
\mathcal{P}^{\square(r)}(x)=\frac{A_{r}(x)+B_{r}(x) \sqrt{1-4 x}}{(1-4 x)^{\gamma_{r}}},
$$

where $A_{r}(x)$ and $B_{r}(x)$ are polynomials and $\gamma_{r}=(3 r-1) / 2$. We find this to be correct for all the cases we have enumerated that is up to $r=5$ :

$$
\begin{aligned}
& 2 A_{2}(x)=x^{2}-26 x^{3}+228 x^{4}-906 x^{5}+1709 x^{6}-1378 x^{7}+322 x^{8}, \\
& 2 B_{2}(x)=-x^{2}+24 x^{3}-182 x^{4}+586 x^{5}-815 x^{6}+404 x^{7}-32 x^{8} \text {. } \\
& A_{3}(x)=x^{2}-22 x^{3}+197 x^{4}-924 x^{5}+2545 x^{6}-5374 x^{7}+13828 x^{8} \\
& -33634 x^{9}+46027 x^{10}-24746 x^{11}+612 x^{12}+256 x^{13}+256 x^{14} \text {, } \\
& B_{3}(x)=-x^{2}+20 x^{3}-159 x^{4}+642 x^{5}-1509 x^{6}+3176 x^{7}-9040 x^{8} \\
& +19254 x^{9}-18943 x^{10}+4968 x^{11}+768 x^{12}+256 x^{13} \text {. } \\
& 2 A_{4}(x)=2 x^{2}-60 x^{3}+809 x^{4}-6564 x^{5}+36321 x^{6}-146436 x^{7} \\
& +439283 x^{8}-960070 x^{9}+1485167 x^{10}-1823356 x^{11} \\
& +2728708 x^{12}-4441406 x^{13}+4054296 x^{14}-932228 x^{15} \\
& -298318 x^{16}-143360 x^{17}+16384 x^{18}-32768 x^{19}, \\
& 2 B_{4}(x)=-2 x^{2}+56 x^{3}-701 x^{4}+5266 x^{5}-26987 x^{6}+100694 x^{7} \\
& -276415 x^{8}+537888 x^{9}-727683 x^{10}+889018 x^{11} \\
& -1536634 x^{12}+2199158 x^{13}-1289388 x^{14}-47472 x^{15} \\
& +26880 x^{16}+50176 x^{17}-6144 x^{18}+8192 x^{19} \text {. } \\
& 2 A_{5}(x)=2 x^{2}-76 x^{3}+1343 x^{4}-14776 x^{5}+114384 x^{6}-666240 x^{7} \\
& +3036602 x^{8}-11071408 x^{9}+32642310 x^{10}-77911156 x^{11} \\
& +148630330 x^{12}-220310536 x^{13}+250700412 x^{14} \\
& -250317844 x^{15}+290657417 x^{16}-309183568 x^{17} \\
& +150313538 x^{18}+21743832 x^{19}-15222464 x^{20}+449152 x^{21} \\
& -3828224 x^{22}-2844672 x^{23}+974848 x^{24}-819200 x^{25} \text {, } \\
& 2 B_{5}(x)=-2 x^{2}+72 x^{3}-1203 x^{4}+12506 x^{5}-91510 x^{6}+504084 x^{7} \\
& -2171612 x^{8}+7467208 x^{9}-20683474 x^{10}+46059704 x^{11} \\
& -80841764 x^{12}+107986392 x^{13}-111525400 x^{14} \\
& +114888220 x^{15}-142562573 x^{16}+122527230 x^{17} \\
& -24478856 x^{18}-17117496 x^{19}-533632 x^{20}-2988544 x^{21} \\
& -808960 x^{22}+401408 x^{23}-819200 x^{24} \text {. }
\end{aligned}
$$

Likewise the generating functions for the $k^{\prime}$ th area moment, $\mathcal{P}_{k}^{\square(r)}(x)$, is also of the form (24)

$$
\mathcal{P}_{k}^{\square(r)}(x)=\frac{A_{r, k}(x)+B_{r, k}(x) \sqrt{1-4 x}}{(1-4 x)^{\gamma_{r+k}}} .
$$


We find that the degrees of the polynomials $A_{r, k}(x)$ and $B_{r, k}(x)$ are less than $5 r+2 k$ for $r \leq 5$ and $k \leq 10$. In particular we have:

$$
\begin{gathered}
\mathcal{P}_{1}^{\square(1)}(x)=\frac{1-14 x+72 x^{2}-162 x^{3}+145 x^{4}-34 x^{5}+2 x^{6}}{(1-4 x)^{5 / 2}} \\
-\frac{1-12 x+50 x^{2}-82 x^{3}+43 x^{4}-4 x^{5}}{(1-4 x)^{2}} .
\end{gathered}
$$

From these solutions we then calculate the exact leading amplitudes and indeed we find that

$$
A_{k}^{(r)}=A_{k, r}\left(x_{c}\right) / \Gamma\left(\gamma_{r+k}\right)=\frac{(-1)^{k+r}(k+r) ! x_{c}^{2 r} f_{k+r}}{r ! x_{c}^{\gamma_{k+r}} \Gamma\left(\gamma_{k+r}\right)}
$$

in complete agreement with Eq. (5).

We have also looked at the amplitudes $B_{k}^{(r)}=B_{k, r}\left(x_{c}\right) / \Gamma\left(\gamma_{r+k}-\frac{1}{2}\right)$ of the correction terms and find, quite remarkably, that they are given simply in terms of the leading amplitudes with one less puncture:

$$
B_{k}^{(r)}=-\frac{1}{8} A_{k}^{(r-1)}
$$

\subsection{Staircase polygons with staircase punctures of fixed size}

Next we examine the more general case of staircase polygons with punctures of fixed size. In the case of one puncture we thus look at staircase polygons with a staircase hole of half-perimeter $s$ while in the case of two punctures we look at staircase polygons with two staircase holes whose half-perimeters sum to $s$. As in the previous section we expect the generating functions to be of the form

$$
\mathcal{P}_{k}^{(r, s)}(x)=\frac{A_{r, s, k}(x)+B_{r, s, k}(x) \sqrt{1-4 x}}{(1-4 x)^{\gamma_{r+k}}} .
$$

We find this to be true with the degree of the polynomials less than $5 r+2(k+s)$.

For once punctured polygons we calculated the generating functions for $s \leq 25$ and $k \leq 10$. In Theorem 1 we proved that the leading amplitude $A_{k}^{(1, s)}=A_{1, s, k}\left(x_{c}\right) / \bar{\Gamma}\left(\gamma_{k+1}\right)=$ $A_{k+1} x_{c}^{s} p_{s}$, where $p_{s}$ is the number of staircase polygons of half-perimeter $s$. This formula is naturally confirmed by our numerical results. Of more interest is the sub-leading amplitude $B_{k}^{(1, s)}$. We find that the result for minimally punctured polygons generalises to this case and $B_{k}^{(1, s)}=-b_{1, s} A_{k}$. We also find that the integer sequence $d_{s}=8^{s-1} b_{1, s}=$ $1,5,29,182, \ldots$ is given by the recurrence:

$$
8 s^{2} d_{s}+(s+3)(7 s+10) d_{s+1}-(s+3)(s+2) d_{s+2}=0, d_{1}=0, d_{2}=1 .
$$

We note that $d_{s}$ grows like $8^{s}$ so that $b_{1, s}$ grows no faster than a polynomial in $s$.

For twice punctured polygons we calculated the generating functions for $s \leq 10$ and $k \leq 10$. As a consequence of theorem 1 the leading amplitude is given by $A_{k}^{(2, s)}=$ $A_{2, s, k}\left(x_{c}\right) / \Gamma\left(\gamma_{k+2}\right)=A_{k+2} x_{c}^{s} \sum_{t=2}^{s-2} p_{s-t} p_{t}$. And again we find that $B_{k}^{(2, s)}=-b_{2, s} A_{k+1}$, though in this case we haven't found a recurrence for the integer sequence $2 \times 8^{s-2} b_{2, s}=$ $1,9,69,510, \ldots$ 


\subsection{Staircase polygons with a single staircase puncture}

We now turn to the analysis of the area moments of staircase polygons with a single staircase puncture of arbitrary size (1-punctured staircase polygons for short). In a recent paper [22] we reported on work which led to an exact Fuchsian linear differential equation of order 8 apparently satisfied by the half-perimeter generating function, $\mathcal{P}^{(1)}(x)=\sum_{m>0} p_{m}^{(1)} x^{m}$, for 1-punctured staircase polygons (that is $\mathcal{P}^{(1)}(x)$ is one of the solutions of the ODE, expanded around the origin). Our analysis of the ODE showed that the dominant singular behaviour is

$$
\mathcal{P}^{(1)}(x) \sim \frac{A(x)}{(1-4 x)}+\frac{B(x)+C(x) \log (1-4 x)}{\sqrt{1-4 x}}+D(x)(1+4 x)^{13 / 2} .
$$

The functions $A(x)-D(x)$ are regular in the disc $|x| \leq 1 / 4$. In addition there were a pair of singularities on the imaginary axis at $x= \pm i / 2$, and at the roots of $1+x+7 x^{2}$. Note that the absolute value of these singularities exceeds $1 / 4$ and so their contributions to the asymptotic growth of the series coefficients are exponentially suppressed.

We expect that the area moment generating functions, $\mathcal{P}_{k}^{(1)}(x)$, should have a similar critical behaviour to that of (30). Indeed our analysis using differential approximants [40] revealed that at $x=x_{c}=1 / 4$ there is a triple root with exponents $-\gamma_{k+1}$ and $-\gamma_{k+1}+1 / 2$ (twice) which is indicative that the behaviour is

$$
\mathcal{P}_{k}^{(1)}(x) \sim \frac{A(x)+[B(x)+C(x) \log (1-4 x)] \sqrt{1-4 x}}{(1-4 x)^{3 k / 2+1}} .
$$

However, the behaviour at the singularity $x=x_{-}=-1 / 4$ is a little more complicated. For the first area moment we find that there is a double root with exponents 5 and $13 / 2$, while for the second area moment we find a triple root at $x_{-}$with exponents $7 / 2,5$ and $13 / 2$. For higher moments the behaviour is consistent with a triple root with exponents $(13-3 k) / 2,(10-3 k) / 2$ and $(7-3 k) / 2$. That is, the value of the leading exponent decreases by $3 k / 2$ and there is in addition a non-analytic correction to scaling with exponent $3 / 2$. The upshot of this analysis is that the asymptotic behaviour of the coefficients of $\mathcal{P}_{k}^{(1)}(x)$ should be given by

$$
\left[x^{m}\right] \mathcal{P}_{k}^{(1)}(x) \sim 4^{m}\left(\sum_{j=0} m^{3 k / 2-j}\left(a_{j}+\frac{1}{\sqrt{m}}\left[b_{j}+c_{j} \log (m)\right]\right)+(-1)^{m} \sum_{j=0} d_{j} m^{(-15+3 k-j) / 2}\right),
$$

where we have ignored the contributions from singularities in the complex plane with absolute values exceeding $1 / 4$. Estimates for the amplitudes were obtained by fitting the coefficients $p_{m}^{(1, k)}=\left[x^{m}\right] \mathcal{P}_{k}^{(1)}(x)$ to the form given above using an increasing number of amplitudes. 'Experimentally' we find we need about the same total number of terms at $x_{c}$ and $x_{-}$. So in the fits we used the terms with amplitudes $a_{i}, b_{i}$ and $c_{i}, i=0, \ldots, K$ and $d_{i}, i=0, \ldots, 3 K$. Going only to $K$ with the $d_{i}$ amplitudes results in much poorer convergence and going beyond $3 K$ leads to no improvement. So we use the $6 K+4$ terms 

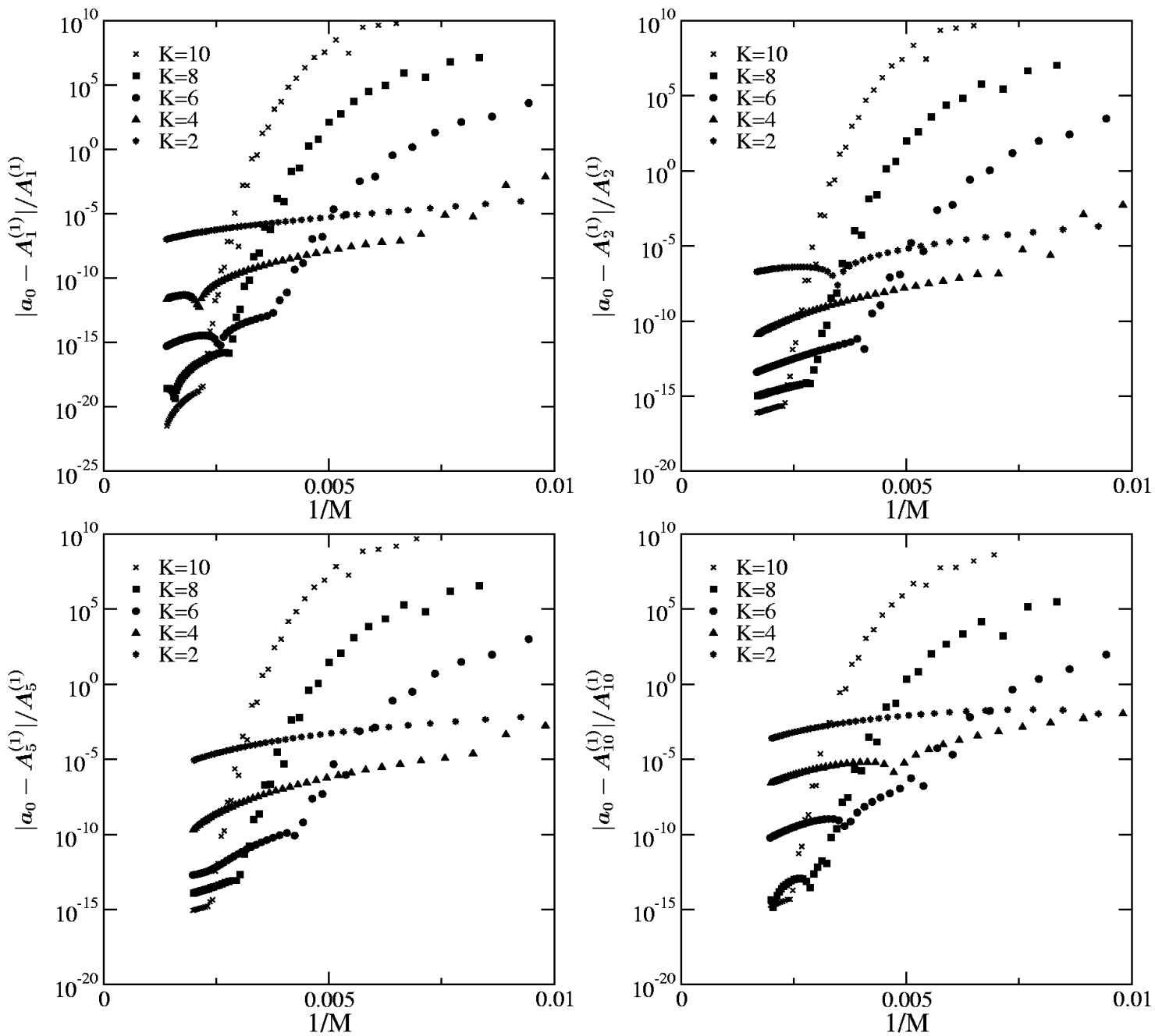

Figure 4: The relative precision of the estimates for the leading amplitude, $\left|a_{0}-A_{k}^{(1)}\right| / A_{k}^{(1)}$, against $1 / M$ for the first (top left panel), second (top right), fifth (bottom left) and tenth (bottom right) area moments. 
$p_{m}^{(1, k)}$ with $m$ ranging from $M$ to $M-6 K-3$ and solve the resulting system of $6 K+4$ linear equations.

We compare the amplitude estimates to the predictions in Section 4.3 and we find that the estimated leading amplitude $a_{0}$ is given by the exact formula

$$
A_{k}^{(r)}=\frac{(-1)^{k+r}(k+r) ! x_{c}^{r} f_{k+r}}{r ! x_{c}^{\gamma_{k+r}} \Gamma\left(\gamma_{k+r}\right)},
$$

which agrees with Eq. (20) since the critical half-perimeter generating, see Eq. (1), for staircase polygons is $\mathcal{P}\left(x_{c}\right)=1 / 4=x_{c}$. In [22] we studied the normalised coefficients $r_{m}=p_{m+8}^{(1)} / 4^{m}$. Using the recurrence relations for $p_{m}^{(1)}$ (derived from the ODE) it is easy and fast to generate many more terms $r_{m}$. We generated the first 100000 terms and saved them as floats with 500 digit accuracy. We found (to better than 100 digits) that the leading amplitude of the normalised series was $\tilde{a}_{0}=1024$. Going back to the normalisation used in this paper we find $a_{0}=1024 / 4^{8}=1 / 64$ in agreement with formula (33). In figure 4 we plot the relative precision of the estimates for the amplitude, $\left|a_{0}-A_{k}^{(r)}\right| / A_{k}^{(r)}$ against $1 / M$ for some of the area moments. Recall that for $k=1$ we have a series of 352 non-zero terms, for $k=2$ we have 292 terms and for $k=3-10$ we have 246 terms. In all cases (including for area moments not shown) the relative precision of the estimate $a_{0}$ is better than $10^{-15}$ for $K=10$. For the first area moment, where we have a longer series, the precision is even more impressive, being better than $10^{-20}$. So in all cases we can confirm the exact prediction (33) for the amplitude to at least 15 significant digits.
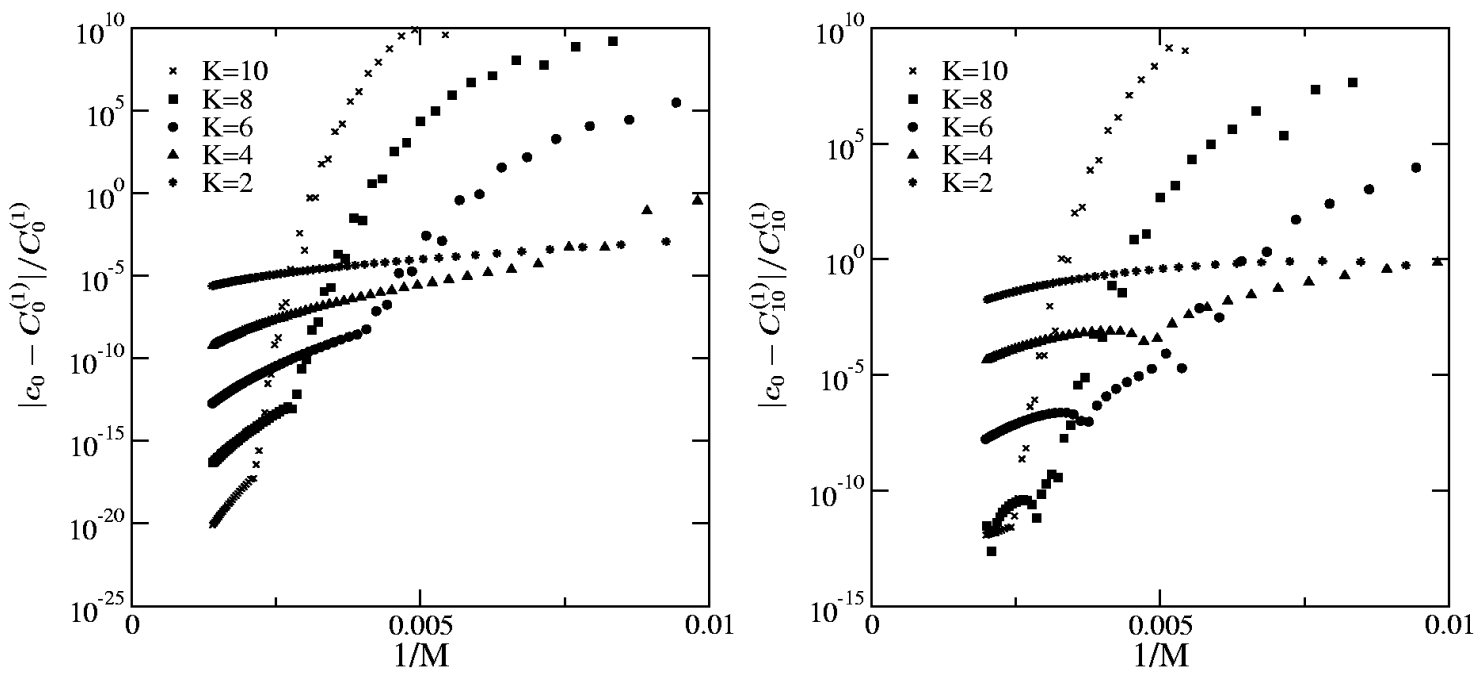

Figure 5: The relative precision of the estimates for the amplitude, $\left|c_{0}-C_{k}^{(1)}\right| / C_{k}^{(1)}$, against $1 / M$ for the zeroth (leftmost panel) and tenth (rightmost panel) area moments.

In the previous section we saw that for minimally punctured staircase polygons the amplitude of the correction term is just a constant times the leading amplitudes with one less puncture, see Eq. (28). It is thus natural to ask if something similar happens 
in the more general case. And indeed we find numerically that the amplitude $C_{k}^{(1)}$ of the dominant correction in (31) (the one proportional to the log-term) giving rise to the $c_{j}$-terms in $(32)$ are

$$
C_{k}^{(1)}=-\frac{3 \sqrt{3}}{8 \pi} A_{k}
$$

In figure 5 we have plotted the relative error between the estimate of $c_{0}$ and the predicted value $C_{k}^{(1)}$ for $k=0$ and 10 . The estimates for the other moments are very similar with the accuracy of the agreement diminishing with higher moments. So in all cases (34) has been confirmed to better than 10 digit accuracy.

For the sub-dominant correction term the amplitude $B_{k}^{(1)}$, as approximated by the term $b_{0}$ in (32), is not simply related to $A_{k}$ and in fact it even changes sign as $k$ is increased.

\subsection{Staircase polygons with two staircase punctures}

In this section we report on our analysis of the series for staircase polygons with two punctures of arbitrary size. Our first task is to work out the singularity structure of the perimeter generating function $\mathcal{P}^{(2)}(x)$ (for which we have a series with 240 nonzero terms). From the general considerations of Section 2 we expect a singularity at $x=x_{c}=1 / 4$ with exponent $-5 / 2$, but given the quite complicated singularity structure of $\mathcal{P}^{(1)}(x)$, as detailed in Eq. (30) and below, we would expect similar complications for $\mathcal{P}^{(2)}(x)$. We analysed the series for $\mathcal{P}^{(2)}(x)$ using differential approximants [40]. This analysis revealed that there is a triple root at $x=x_{c}=1 / 4$ and the exponents had values $-2.499(1),-2.070(5)$ and $-1.78(1)$. So despite having a series of 240 terms it is still very difficult to pin down the exponents accurately. Given the values quoted above two possible scenarios present themselves. Either the exponents have the exact values $-5 / 2$, -2 and -2 or they have the exact values $-5 / 2,-2$ and $-7 / 4$. The behaviour of $\mathcal{P}^{(1)}(x)$ would support the first of these scenarios and below we shall present evidence from the analysis of the asymptotic form of the coefficients which strongly supports this behaviour. We also find a double root singularity at $x=x_{-}=-1 / 4$ with exponent estimates consistent with the exact values 5 and $11 / 2$. In addition there are several conjugate pairs of singularities in the complex plane. The most important of these are at $x=(-1 \pm i \sqrt{3}) / 8$, which has magnitude $1 / 4$ and thus lies equidistant from the origin to $x_{c}$. So unlike the situation for once punctured staircase polygons we cannot ignore the complex singularities. The exponent estimate at this singularity is consistent with the exact value $33 / 2$. The singularities at $x=(-1 \pm i \sqrt{3}) / 8$ are the roots of the polynomial $1+4 x+16 x^{2}$ which would indicate that the generating function contains a term $\sim E(x)\left(1+4 x+16 x^{2}\right)^{33 / 2}$. Finally, we find some singularities with magnitude greater than $1 / 4$. There are singularities at $x=(1 \pm i \sqrt{3}) / 6$ (which has magnitude $1 / 3$ ) with an exponent $33 / 2$ (we note that these are the roots of $\left(1-3 x+9 x^{2}\right)$ ) and at $x= \pm i / 2$ (magnitude $1 / 2$ ) with an exponent consistent with the value 5 . We also find weak evidence that $\mathcal{P}^{(2)}(x)$, just as $\mathcal{P}^{(1)}(x)$, has a pair of singularities at the roots of $1+x+7 x^{2}$.

As noted above we need to include the contribution from a conjugate pair of complex 
singularities to the asymptotic form of the coefficients. In general this is not as straightforward a task as dealing with singularities on the real axis. In [41] we examined the generating function of self-avoiding walks on the honeycomb lattice which has a pair of singularities on the imaginary axis at $x= \pm i / \tau$, arising from a term of the form $H(x)\left(1+\tau^{2} x^{2}\right)^{-\eta}$. This typically produces coefficients which change sign according to a ++-- pattern. This can be accommodated by including terms of the form

$$
\sim \tau^{m} m^{\eta-1} \sum_{j \geq 0}(-1)^{\lfloor(m+j) / 2\rfloor} h_{j} / m^{j}
$$

in fitting to the asymptotic form of the coefficients.

Note that the analysis in [41] clearly demonstrated that, as is done above, one has to shift the sign-pattern by $j$ when terms proportional to $1 / m^{j}$ are considered. Terms arising from other complex conjugate pairs of singularities can give rise to much more complicated sign-patterns. In order to handle such cases we simply form the Taylor expansion of the simplest possible term arising from the singularity and take the sign of the appropriate coefficient. Specifically in order to include terms proportional to $1 / \mathrm{m}^{j}$, when looking at the coefficients $\left[x^{m}\right] \mathcal{P}^{(2)}(x)$, we take the sign to be the sign of the coefficient of $x^{m+j}$ in the Taylor expansion of the function $\left(1+4 x+16 x^{2}\right)^{33 / 2}$. We use the notation Sign $\left(\left[x^{m+j}\right]\left(1+4 x+16 x^{2}\right)^{33 / 2}\right)$ for this operation.

The singularity structure revealed above leads us to fit the coefficients to a form, which is appropriate if the first scenario (exponents at $x=x_{c}=1 / 4$ are $-5 / 2,-2$ and -2 ) is correct

$$
\begin{gathered}
{\left[x^{m}\right] \mathcal{P}^{(2)}(x)=4^{m}\left(\sum_{j=0}^{K} m^{3 / 2-j}\left[a_{j}+\frac{1}{\sqrt{m}}\left[b_{j}+c_{j} \log (m)\right]\right]+(-1)^{m} \sum_{j=0}^{3 K} d_{j} m^{-6-j / 2}\right.} \\
\left.+\sum_{j=0}^{3 K} e_{j} \operatorname{Sign}\left(\left[x^{m+j}\right]\left(1+4 x+16 x^{2}\right)^{33 / 2}\right) m^{-35 / 2+j}\right)
\end{gathered}
$$

where we have ignored the singularities with magnitude exceeding $1 / 4$. We also examine the alternative form appropriate if the second scenario (exponents at $x=x_{c}=1 / 4$ are $-5 / 2,-2$ and $-7 / 4)$ is correct

$$
\begin{aligned}
{\left[x^{m}\right] \mathcal{P}^{(2)}(x)=4^{m} } & \left(\sum_{j=0}^{K} m^{3 / 2-j}\left[a_{j}+\frac{b_{j}}{m^{1 / 2}}+\frac{c_{j}}{m^{3 / 4}}\right]+(-1)^{m} \sum_{j=0}^{3 K} d_{j} m^{-6-j / 2}\right. \\
& \left.+\sum_{j=0}^{3 K} e_{j} \operatorname{Sign}\left(\left[x^{m+j}\right]\left(1+4 x+16 x^{2}\right)^{33 / 2}\right) m^{-35 / 2+j}\right) .
\end{aligned}
$$

In figure 6 we have plotted the resulting estimates for the leading amplitude $a_{0}$, which we expect are given by the exact value $A_{0}^{(2)}=\frac{5}{3072 \sqrt{\pi}}$, and the amplitude $c_{0}$ of the subdominant terms from the two alternative asymptotic forms. First we focus on the subdominant terms. In the top right panel we show the estimates when fitting to the form (35) where we have a sub-dominant term proportional to $m \log (m)$ while the bottom right panel shows the estimates obtained when fitting to the form (36) where the sub-dominant term is $\mathrm{m}^{3 / 4}$ (in both cases the dominant term is proportional to $\mathrm{m}^{3 / 2}$ with a second sub-dominant term proportional to $m$ ). In the bottom right panel we note that the estimates for the amplitude $c_{0}$ of the term $\mathrm{m}^{3 / 4}$ seems to diverge. As $K$ is increased rather than settle down 

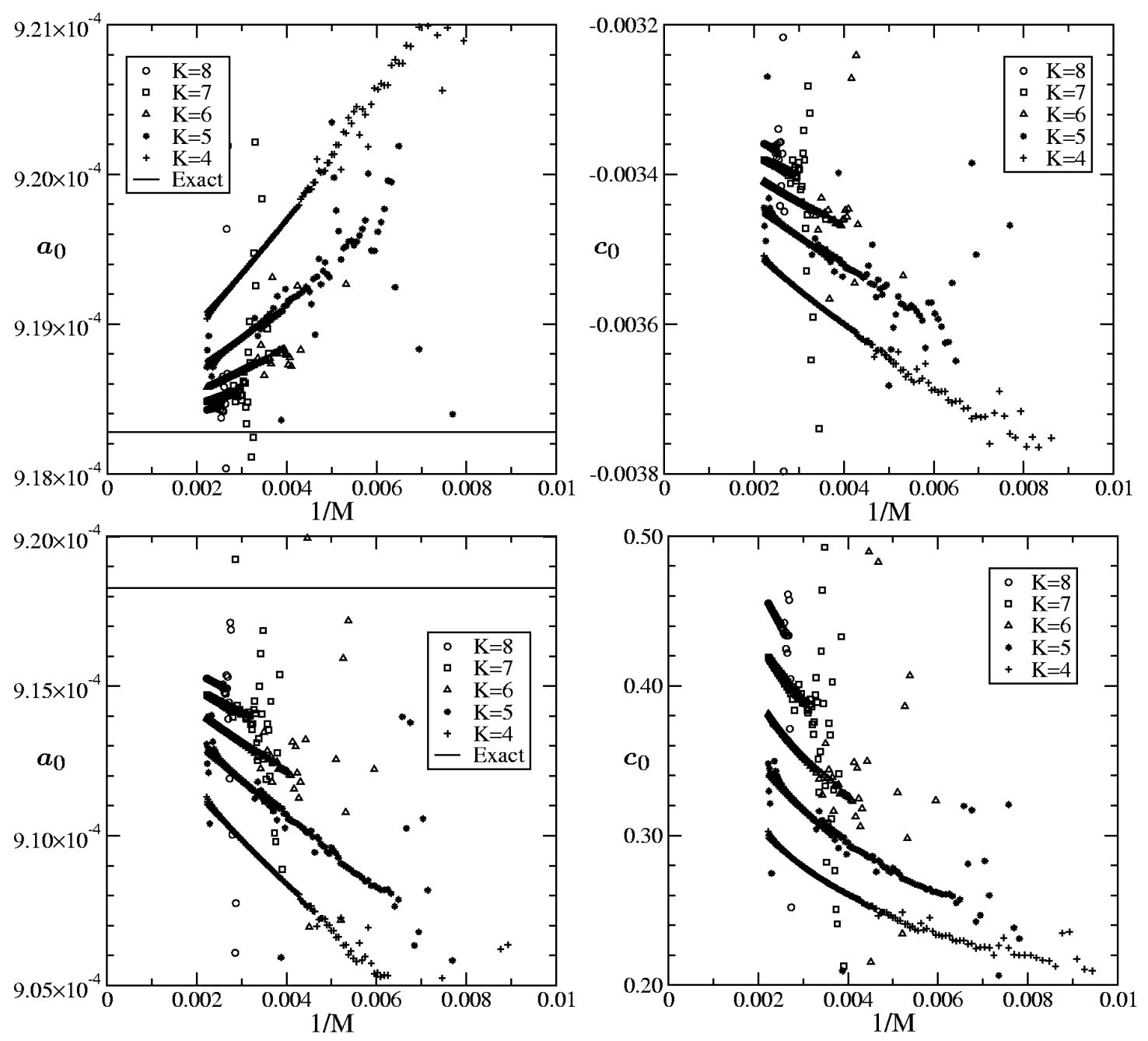

Figure 6: Estimates of the leading amplitude $a_{0}$ and the amplitude $c_{0}$ of the sub-dominant term. The top panels show the results from fitting to the form (35) while the bottom panels are results from fitting to the form (36). The straight line is the exact value of this amplitude $A_{0}^{(2)}$ 
we find that the slope of the curves of the estimates plotted vs. $1 / M$ increases which is the opposite of what we would expect if we are fitting to the correct asymptotic form. We take this as firm numerical evidence that (36) is incorrect. This could also explain why the corresponding estimates of $a_{0}$ (bottom left panel) don't appear to converge to the predicted exact value. In contrast the estimates for $c_{0}$ of the term $m \log (m)$ (top right panel) from the form (35) do seem to converge and the slopes of the estimates plotted vs. $1 / M$ decrease with $K$. In this case the estimates for $a_{0}$ (top left panel) clearly can be extrapolated to a value consistent with the predicted exact value. Note further that the top left panel has a resolution along the ordinate which is a factor 5 higher than in lower right panel so the estimates when fitting to the form (35) are much more tightly converged. The conclusion is that the numerical evidence clearly favours the asymptotic form (35) and we believe this to be (if not entirely correct at least a very good approximation to) the true asymptotic form of the coefficients of the generating function $\mathcal{P}^{(2)}(x)$ for twice punctured staircase polygons.

Now that we have settled the question of the correct singularity structure of $\mathcal{P}^{(2)}(x)$ we turn our attention to the analysis of the area moment generating function $\mathcal{P}_{k}^{(2)}(x)$. As for once punctured staircase polygons we find that the leading exponent at all singularities decreases by $3 k / 2$. So in order to estimate the leading amplitude $a_{0}$ we fit to the form

$$
\begin{aligned}
& {\left[x^{m}\right] \mathcal{P}_{k}^{(2)}(x)=4^{m}\left(\sum _ { j = 0 } ^ { K } m ^ { 3 ( 1 + k ) / 2 - j } \left[a_{j}+\frac{1}{\sqrt{m}}\left[b_{j}+c_{j} \log (m)\right]+\right.\right.} \\
& \left.+(-1)^{m} \sum_{j=0}^{3 K} d_{j} m^{(-12+3 k+j) / 2}+\sum_{j=0}^{3 K} e_{j} \operatorname{Sign}\left(\left[x^{m+j}\right]\left(1+4 x+16 x^{2}\right)^{33 / 2}\right) m^{-(35-3 k+j) / 2}\right)
\end{aligned}
$$

where for simplicity our notation suppresses the $k$-dependence of the amplitudes. Recall that for $k=1$ and 2 we have series with 214 terms and for $k=3-10$ we have 140 terms. We compare the amplitude estimates to the predictions of Section 4. The leading amplitude $a_{0}=A_{k}^{(2)}$ is given by the exact formula (33). In figure 7 we show the estimates for the leading amplitudes for area moments with $k=1,2,5$, and 10. In all cases the amplitudes estimates appears to converge to the predicted exact value and agreement is found to at least 3 significant digits.

Finally we turn our attention to the amplitude $C_{k}^{(2)}$ of the dominant correction term. In the leftmost panel of figure 8 we have plotted the ratio between the estimated amplitude $c_{0}$ and the predicted value of $A_{k}^{(1)}$ for $k=0$ using several cut-offs. In the rightmost panel we show the same ratio but for several different moments using the cut-off $K=6$. These plots are consistent with $C_{k}^{(2)} \propto A_{k}^{(1)}$ with the constant of proportionality being $-0.212(2)$.

\subsection{Punctured self-avoiding polygons}

Before proceeding to the estimation of the amplitudes we briefly have a look at the critical behaviour of the area moment generating functions for punctured self-avoiding polygons. In [16] we analysed the behaviour of $\mathcal{P}_{k}^{(r)}(x)$ and found the critical behaviour 

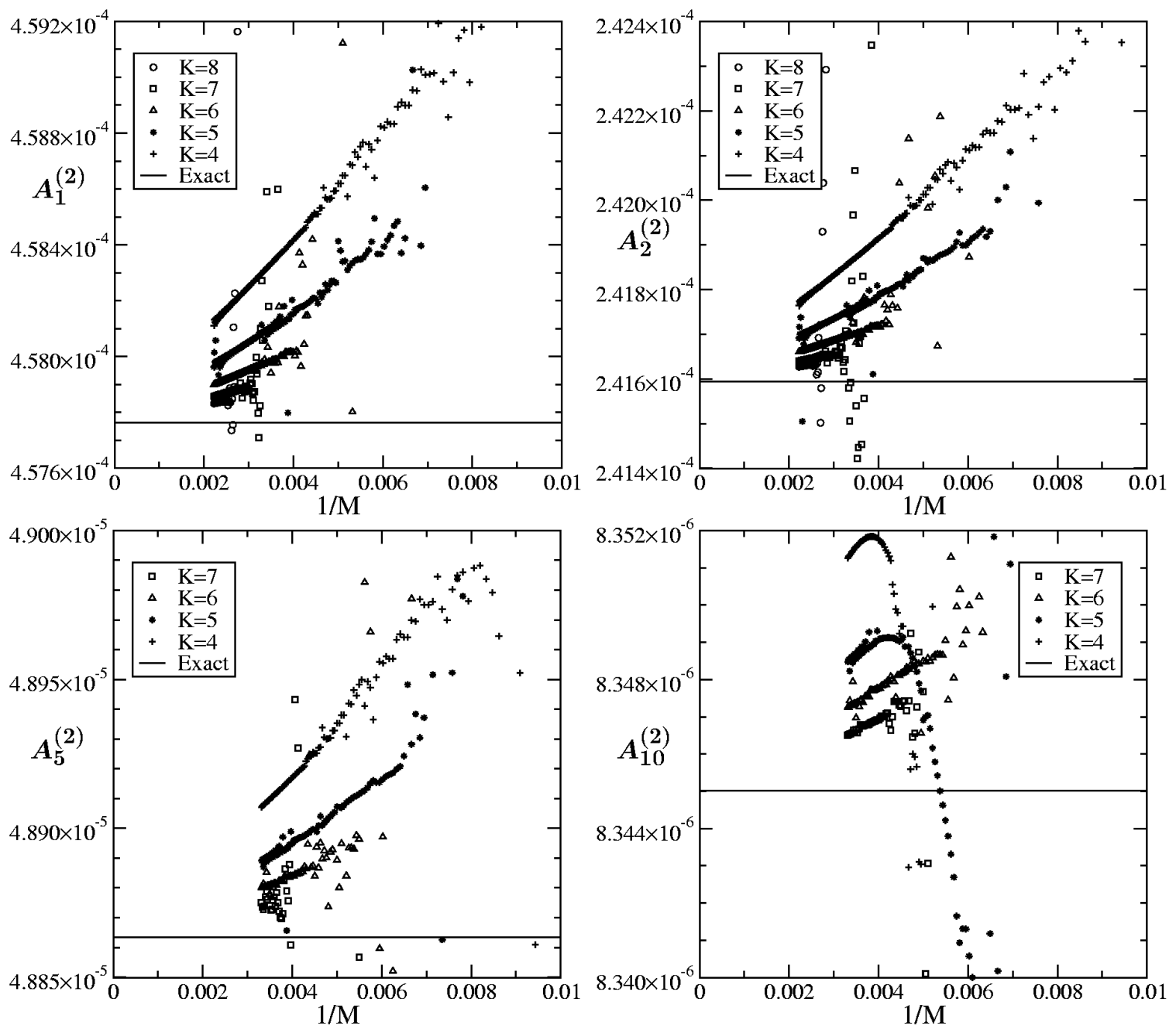

Figure 7: Estimates of the leading amplitude $A_{k}^{(2)}$ of various area moments obtained by fitting to the form (37). The straight line is the exact value of this amplitude.

\begin{tabular}{llll}
\hline \hline \multicolumn{1}{c}{ Degrees } & \multicolumn{3}{c}{ Once punctured $k=0$} \\
\hline$[8,9,9,10]$ & $-0.002022-0.023965 i$ & $-0.002022+0.023965 i$ & 0.518101 \\
{$[8,9,9,11]$} & $-0.003847+0.034768 i$ & $-0.003847-0.034768 i$ & 0.528973 \\
{$[8,9,9,12]$} & $-0.004553-0.038104 i$ & $-0.004553+0.038104 i$ & 0.533388 \\
{$[8,9,10,10]$} & $-0.001830-0.023143 i$ & $-0.001830+0.023143 i$ & 0.515916 \\
{$[8,9,10,11]$} & -0.044131 & 0.060194 & 0.459558 \\
{$[8,9,11,10]$} & $-0.006365-0.045090 i$ & $-0.006365+0.045090 i$ & 0.547479 \\
{$[8,10,9,10]$} & $-0.007054-0.047578 i$ & $-0.007054+0.047578 i$ & 0.552445 \\
{$[8,10,9,11]$} & $-0.009398-0.055135 i$ & $-0.009398+0.055135 i$ & 0.570859 \\
{$[8,10,10,10]$} & $-0.012495-0.063935 i$ & $-0.012495+0.063935 i$ & 0.595957 \\
\hline \hline
\end{tabular}

Table 2: Biased estimates of the critical exponents of once punctured SAPs. 

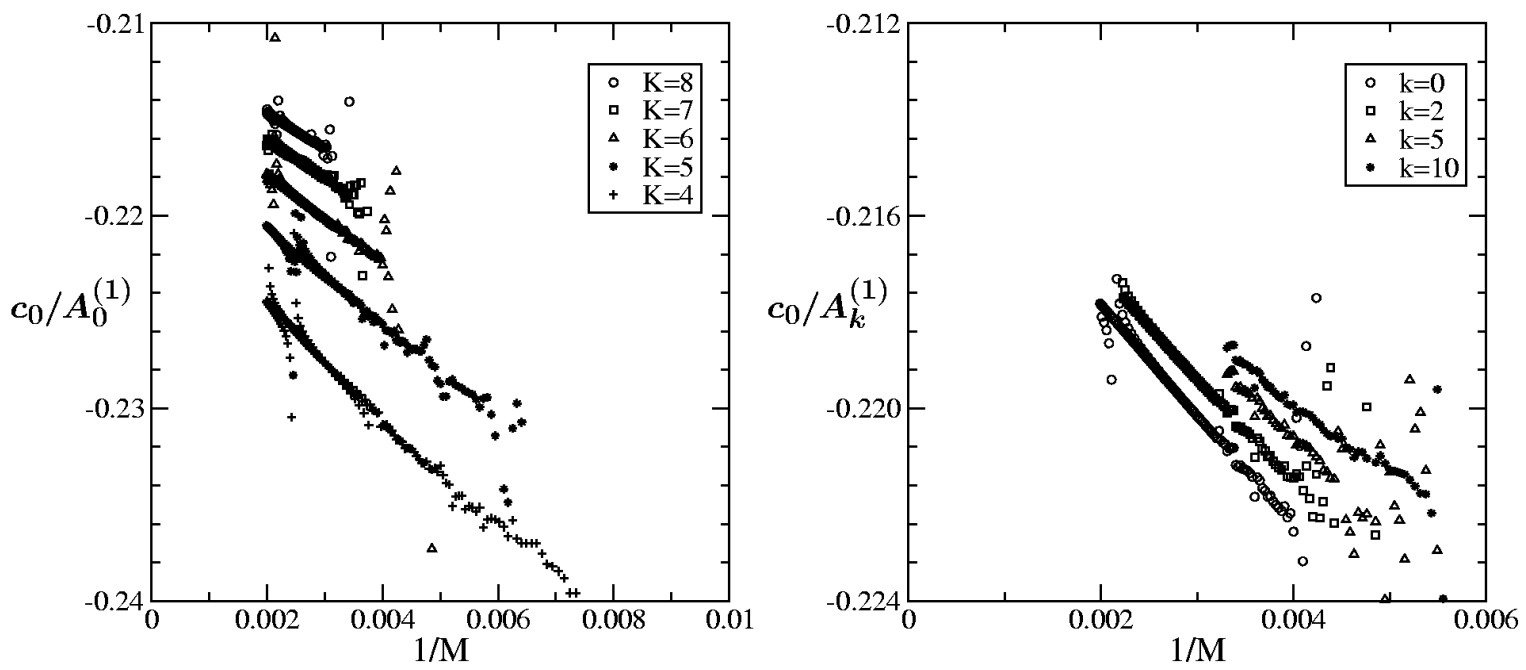

Figure 8: The ratio $c_{0} / A_{k}^{(1)}$ for the zeroth area moment (leftmost panel) and several different area moments (rightmost panel).

to be consistent with $\mathcal{P}_{k}^{(r)}(x) \sim A(x)+B(x)\left(x-x_{c}\right)^{-\gamma_{k+r}}+C(x)\left(x-x_{c}\right)^{-\gamma_{k+r}+1 / 2}$, where $\gamma_{j}=3 j / 2-3 / 2$. From previous work $[42,38]$ we have very precise estimates for $x_{c}$, which is indistinguishable from the positive root of the polynomial $581 x^{2}+7 x-13$, that is, $x_{c}=0.1436806292 \ldots$. Using this value for $x_{c}$ we form a $K^{t h}$-order biased differential approximant $(\mathrm{DA})$ to $\mathcal{P}_{k}^{(r)}(x)$ by matching the coefficients in the polynomials $Q_{i}(x)$ of degree $N_{i}$ so that (one) of the formal solutions to the homogeneous differential equation

$$
\sum_{i=0}^{K}\left(x-x_{c}\right)^{i} Q_{i}(x)\left(x \frac{\mathrm{d}}{\mathrm{d} x}\right)^{i} \tilde{P}(x)=0
$$

agrees with the first $M=\sum_{i}\left(N_{i}+1\right)$ series coefficients of $\mathcal{P}_{k}^{(r)}(x)$. We are thus 'forcing' the DAs to have regular singular points at the origin and $x_{c}$. The critical exponents $\gamma_{j}$ $(j=1 \ldots K)$ are estimated from the indicial equation at $x_{c}$ (note that due to the biasing, $x_{c}$ is root of order $K$ ). If the true singular behaviour at $x_{c}$ implies a root of degree less than $K$ we expect that the 'true' exponents will be quite well estimated and show little scatter while the 'surplus' exponents will show a lot of random scatter. In the following we always use $K=3$ and denote the degrees of the polynomials $Q_{i}$ as $\left[N_{3}, N_{2}, N_{1}, N_{0}\right]$.

First we look at the perimeter generating function for once punctured SAPs (for which we have series with 42 terms). In this case we have $\gamma_{1}=0$ and we thus expect a logarithmic singularity at $x_{c}$ with a square-root correction term as argued in [16]. In Table 2 we list some exponent estimates obtained from the biased DAs. The exponent estimates are indeed consistent with the exact values $0,0,1 / 2$, which confirms the expected behaviour.

In Table 3 we list some exponent estimates for the 2nd and 5th area moments of once punctured SAPs. The exponents support the expectations for the leading and subdominant exponent. The third exponent is of no significance-the nature of the differential approximant forces a third exponent to have some value, but its lack of convergence 


\begin{tabular}{lllllll}
\hline \hline \multicolumn{1}{c}{ Degrees } & \multicolumn{3}{c}{ Once punctured $k=2$} & \multicolumn{3}{c}{ Once punctured $k=5$} \\
\hline$[8,9,9,10]$ & -3.000291 & -2.493921 & -1.154513 & -7.500838 & -6.976201 & -5.107975 \\
{$[8,9,9,11]$} & -3.000158 & -2.496645 & -1.258606 & -7.503030 & -6.927432 & -2.422194 \\
{$[8,9,9,12]$} & -3.012422 & -2.421400 & -3.323742 & -7.500231 & -6.992184 & -5.461825 \\
{$[8,9,10,10]$} & -2.999849 & -2.502858 & -1.447159 & -7.517118 & -6.776728 & -8.010389 \\
{$[8,9,10,11]$} & -3.000452 & -2.491160 & -1.022360 & -7.500700 & -6.980331 & -5.251153 \\
{$[8,9,11,10]$} & -3.000143 & -2.497148 & -1.307314 & -7.500366 & -6.988804 & -5.406296 \\
{$[8,10,9,10]$} & -2.999481 & -2.511762 & -1.668527 & -7.505629 & -6.879658 & -14.20958 \\
{$[8,10,9,11]$} & -2.999906 & -2.501910 & -1.441981 & -7.500535 & -6.984576 & -5.361394 \\
{$[8,10,10,10]$} & -2.999647 & -2.507601 & -1.578352 & -7.500946 & -6.974159 & -5.059683 \\
\hline \hline
\end{tabular}

Table 3: Biased estimates of the critical exponents for the 2nd and 5th area moments of once punctured SAPs.

\begin{tabular}{lllrrrr}
\hline \hline \multicolumn{1}{c}{ Degrees } & \multicolumn{3}{c}{ Twice punctured $k=0$} & \multicolumn{3}{c}{ Twice punctured $k=2$} \\
\hline$[7,8,8,9]$ & -1.504250 & -0.968766 & 6.594627 & -4.499111 & -4.006801 & -2.544930 \\
{$[7,8,8,10]$} & -1.504232 & -0.968829 & 5.029521 & -4.495871 & -4.039199 & -3.083192 \\
{$[7,8,8,11]$} & -1.504209 & -0.968910 & -60.90721 & -4.494258 & -4.048789 & -2.701322 \\
{$[7,8,9,9]$} & -1.504210 & -0.968909 & 12.03204 & -4.494568 & -4.047486 & -2.836670 \\
{$[7,8,9,10]$} & -1.504197 & -0.968951 & 32.28205 & -4.493872 & -4.051402 & -2.672813 \\
{$[7,8,10,9]$} & -1.504205 & -0.968923 & 22.27646 & -4.508207 & -3.915977 & -1.950665 \\
{$[7,9,8,9]$} & -1.504178 & -0.969017 & 18.18298 & -4.495340 & -4.040712 & -2.812621 \\
{$[7,9,8,10]$} & -1.504177 & -0.969020 & 18.81517 & -4.495136 & -4.041237 & -2.679530 \\
{$[7,9,9,9]$} & -1.504176 & -0.969022 & 18.58757 & -4.498396 & -4.012876 & -2.698591 \\
\hline \hline
\end{tabular}

Table 4: Biased estimates of the critical exponents for the 0th and 2nd area moments of twice punctured SAPs. 
suggests it is not, in fact present. In Table 4 we list exponent estimates for the 0th and 2nd area moments of twice punctured SAPs (for which we have series with 38 terms). Similar comments apply to the three columns of exponent estimates as were made for the 2 nd area moments. In all cases we get a clear confirmation of the critical behaviour observed in [16].

Next we turn our attention to estimates for the critical amplitudes. Proposition 2 and Theorem 2 tells us that the critical amplitude of the $k$ th area moment of self-avoiding polygons with $r$ (minimal or arbitrary) punctures is proportional to the critical amplitude of the $(k+r)$ th area moment of unpunctured SAPs (the theorems also give the constants of proportionality). In order to test these predictions numerically we analyse in this section data for SAPs with one and two punctures. In all cases we look at the ratio $r_{m}=p_{m}^{(r, k)} / p_{m}^{(k+r)}$ which should approach the relevant constant of proportionality. Given the critical behaviour outlined above we expect that these amplitude ratios can be approximated quite well by the asymptotic form

$$
r_{m}=\sum_{j=0} a_{j} m^{j / 2}
$$

So as in the analysis of punctured staircase polygons we obtain estimates for the leading amplitude $a_{0}$, by fitting to the above form truncated at some level $K$, and we then plot these estimates against $1 / M$.

\subsubsection{Minimal punctures}

According to Proposition 2 the amplitude of the $k$-th area moment of SAPs with $r$ minimal punctures is

$$
A_{k}^{(r)}=A_{k+r} x_{c}^{2 r} / r !
$$

We first analyse the area moments of SAPs with a single minimal puncture. In the left panel of figure 9 we have plotted estimates for amplitude ratio $A_{0}^{(1)} / A_{1}$ with $7 \leq$ $K \leq 10$. The prediction for this ratio is $x_{c}^{2}$, which is plotted as a straight line. The estimates obtained with $K=9$ and 10 are indistinguishable from the predicted value at the resolution of the plot. Note that the 'curvature' of the plotted values decreases as $K$ increases. We take this to be a very clear indication that the ratio $r_{m}$ is very well approximated by the assumed asymptotic form. In the right panel we plot the estimates for the amplitude ratio $A_{k}^{(1)} / A_{k+1}$ for $0 \leq k \leq 9$ using the cut-off value $K=10$. For small values of $k$ these plots give firm numerical evidence for the correctness of Proposition 2. For higher values of $k$ ( 8 and 9 in particular) the evidence is not quite as firm though nothing in the plot would suggest a discrepancy with the predicted value. We again emphasise that in this case we have relatively short series of only 42 terms.

Next we analyse the area moments of SAPs with two minimal punctures. The left panel of figure 10 shows estimates for amplitude ratio $A_{0}^{(2)} / A_{2}$ with $7 \leq K \leq 10$. The prediction for the ratio, $x_{c}^{4} / 2$, is plotted as a straight line. Again we find that the estimates for $K=10$ are indistinguishable from the predicted value, though in this case the relative 

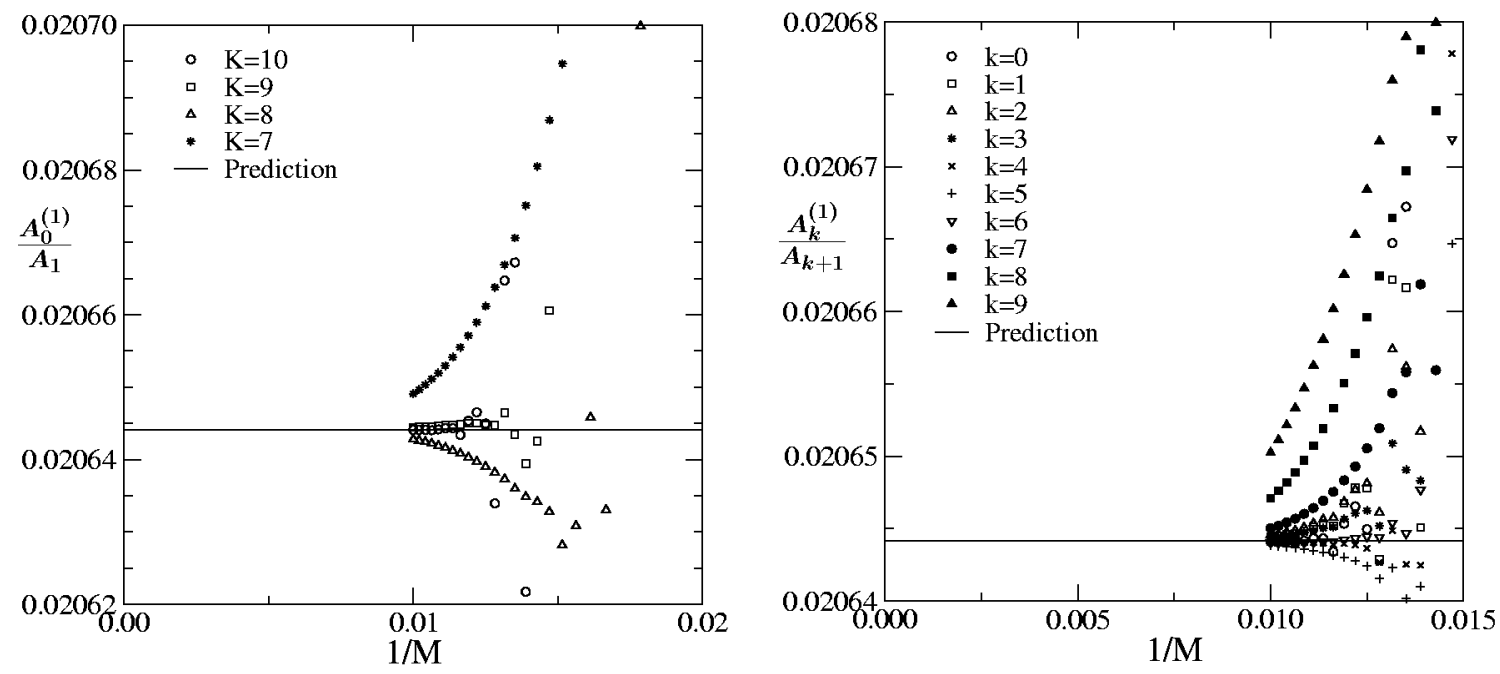

Figure 9: Estimates of the amplitude ratio $A_{k}^{(1)} / A_{k+1}$ for self-avoiding polygons with one minimal puncture.

resolution is coarser than in the previous plot. In the right panel we plot estimates of the amplitude ratio $A_{k}^{(2)} / A_{k+2}$ for $0 \leq k \leq 8$ using the cut-off value $K=10$. Again we find that our numerical analysis confirms the prediction to a high degree of confidence. Recall that in this case we have series with only 38 terms.

\subsubsection{Arbitrary punctures}

According to Theorem 2 the amplitude of the $k$-th area moment of SAPs with $r$ arbitrary punctures is

$$
A_{k}^{(r)}=A_{k+r}\left(\mathcal{P}\left(x_{c}\right)\right)^{r} / r !
$$

where $\mathcal{P}\left(x_{c}\right)$ is the critical amplitude of the half-perimeter generating function.

The first step in our analysis is to obtain an accurate estimate of $\mathcal{P}\left(x_{c}\right)$. In [42] we obtained the rather imprecise estimate $\mathcal{P}\left(x_{c}\right) \approx 0.036$ by evaluating Padé approximants to the generating function. Here we shall estimate $\mathcal{P}\left(x_{c}\right)$ directly from the perimeter data. We first tried the form

$$
\sum_{m=0}^{M} p_{m} x_{c}^{m} \sim \mathcal{P}\left(x_{c}\right)+a_{1} / M^{1 / 2}+a_{2} / M+\cdots
$$

Using the first ten terms in this asymptotic expansion we found (with $M=55$ ) that $\mathcal{P}\left(x_{c}\right) \approx 0.0362642$, but $a_{1} \approx 4.28 \times 10^{-9}$ and $a_{2} \approx-1.42^{-7}$, while $a_{3} \approx-0.066$. We are therefore quite confident that $a_{1}=a_{2}=0$. Upon further analysis we found convincing evidence that the correct asymptotic form in fact is 

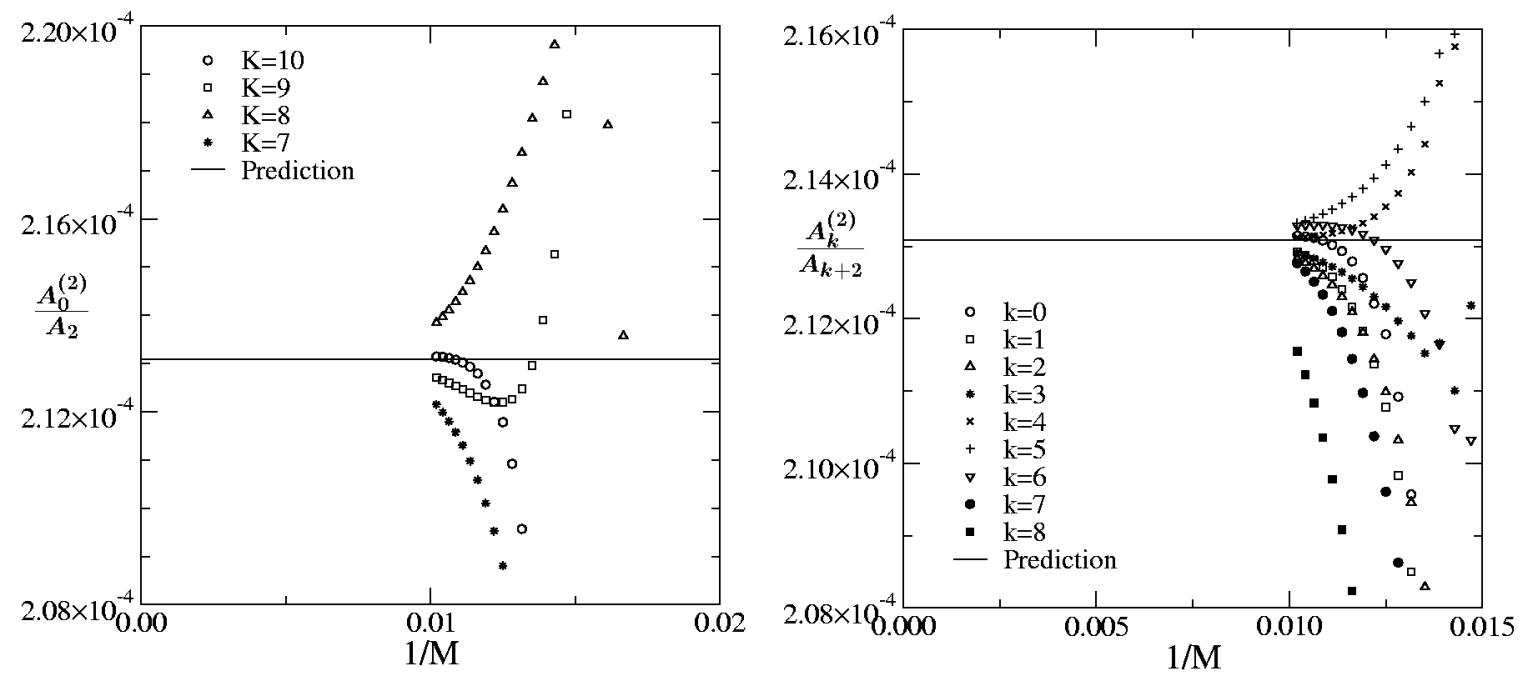

Figure 10: Estimates of the amplitude ratio $A_{k}^{(2)} / A_{k+2}$ for self-avoiding polygons with two minimal punctures.

$$
\sum_{m=0}^{M} p_{m} x_{c}^{m} \sim \mathcal{P}\left(x_{c}\right)+b_{1} / M^{3 / 2}+b_{2} / M^{5 / 2}+\cdots
$$

Indeed, the observation that $a_{1}$ and $a_{2}$ vanish follows from the assumed asymptotic behaviour of $p_{m}$. In Table 5 we have listed estimates for $\mathcal{P}\left(x_{c}\right)$ obtained using various values of $M$ and the cut-off $K$ in the asymptotic form. From this we confidently estimate that $\mathcal{P}\left(x_{c}\right)=0.0362642151808(2)$.

In figure 11 we have plotted estimates of the amplitude ratio $A_{k}^{(1)} / A_{k+1}$ for self-avoiding polygons with one puncture of arbitrary size. In the leftmost panel we look at the ratio $A_{0}^{(1)} / A_{1}$ using different cut-offs $K$. The rightmost panel shows the ratio $A_{k}^{(1)} / A_{k+1}$ for area moments up to $k=9$ using the cut-off $K=10$. The straight line corresponds to the expected value $\mathcal{P}\left(x_{c}\right)$, using the estimate for this quantity obtained above. The estimates in the leftmost panel show some variation when plotted against $1 / M$, but in the limit $M \rightarrow \infty$ the estimates appear to converge to the expected value (if the trend holds). As for minimally punctured SAPs we see an ever closer agreement as $K$ is increased, again indicating that the assumed asymptotic form is reasonable. Obviously, as seen in the rightmost panel, the estimates for high area moments are not as close to the expected value. However, given the trend in these estimates we are confident in stating that our numerical analysis is consistent with the results of Theorem 2. The agreement is particularly impressive bearing in mind that we analyse series with just 42 terms.

Finally in figure 12 we have plotted our estimates of the amplitude ratio $A_{k}^{(2)} / A_{k+2}$ for self-avoiding polygons with two punctures of arbitrary size. The straight line corresponds to the expected value $\mathcal{P}\left(x_{c}\right)^{2} / 2$. Again all estimates are consistent with the results of Theorem 2, though the numerical agreement is less convincing, but then again the series 


\begin{tabular}{cccc}
\hline \hline$M$ & $K=8$ & $K=10$ & $K=12$ \\
\hline 45 & 0.036264215181387 & 0.036264215181095 & 0.036264215180475 \\
46 & 0.036264215181343 & 0.036264215181088 & 0.036264215181354 \\
47 & 0.036264215181305 & 0.036264215181073 & 0.036264215180915 \\
48 & 0.036264215181271 & 0.036264215181060 & 0.036264215180994 \\
49 & 0.036264215181240 & 0.036264215181048 & 0.036264215180970 \\
50 & 0.036264215181214 & 0.036264215181038 & 0.036264215181001 \\
51 & 0.036264215181190 & 0.036264215181029 & 0.036264215180972 \\
52 & 0.036264215181168 & 0.036264215181021 & 0.036264215180972 \\
53 & 0.036264215181149 & 0.036264215181013 & 0.036264215180969 \\
54 & 0.036264215181131 & 0.036264215181006 & 0.036264215180967 \\
55 & 0.036264215181116 & 0.036264215181000 & 0.036264215180962 \\
\hline \hline
\end{tabular}

Table 5: Estimates of the critical SAP amplitude $\mathcal{P}\left(x_{c}\right)$.
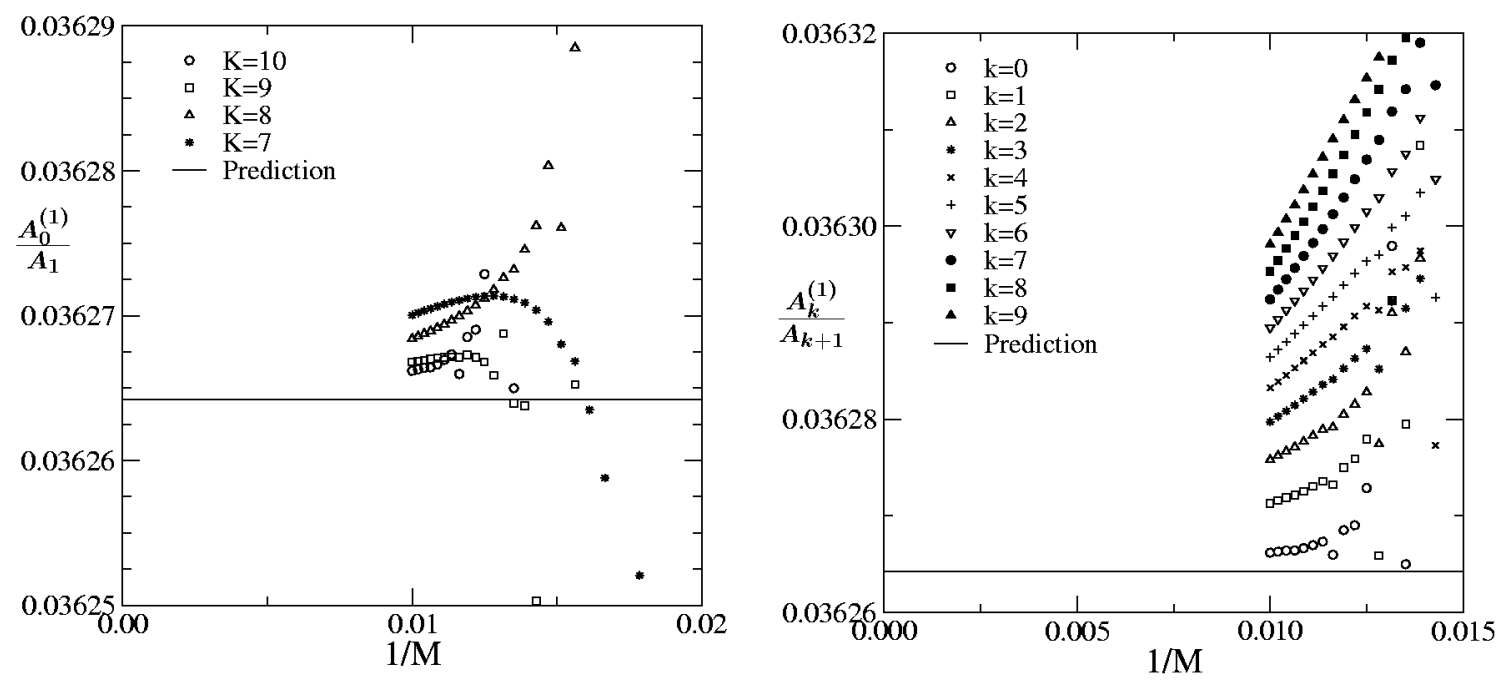

Figure 11: Estimates of the amplitude ratio $A_{k}^{(1)} / A_{k+1}$ for self-avoiding polygons with one puncture of arbitrary size. 

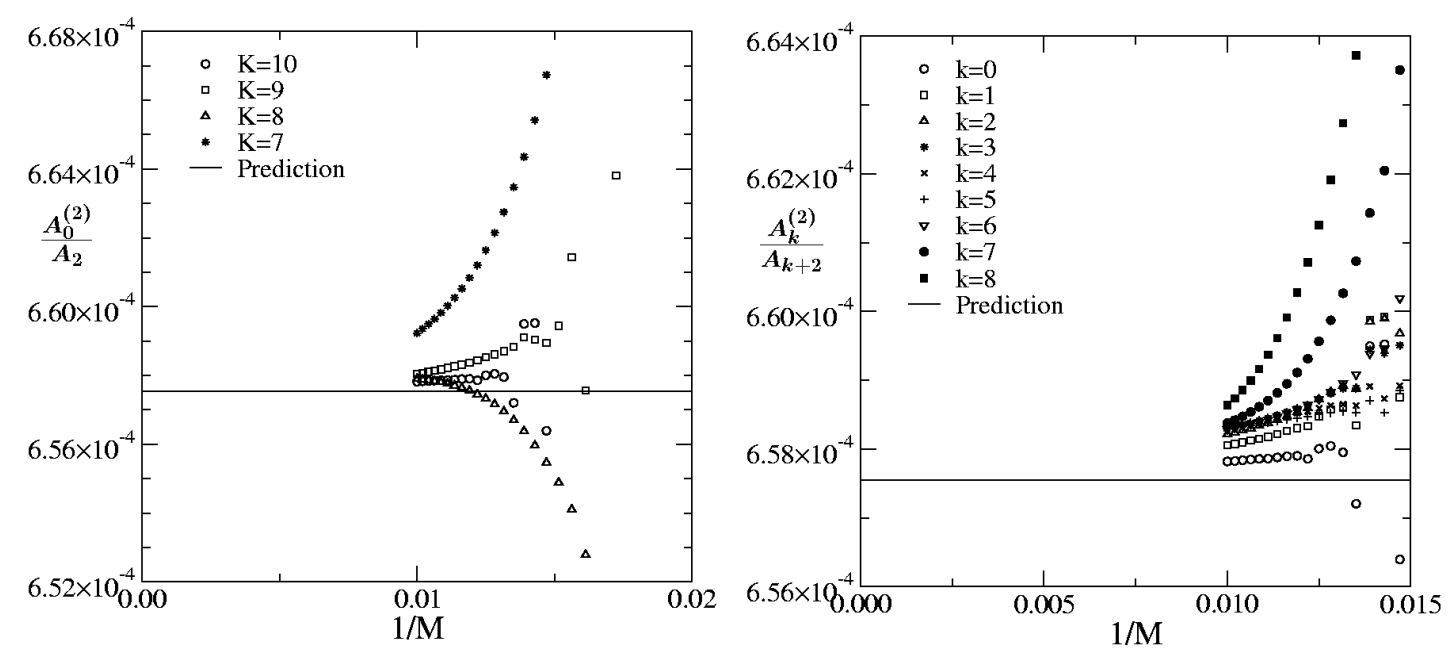

Figure 12: Estimates of the amplitude ratio $A_{k}^{(2)} / A_{k+2}$ for self-avoiding polygons with two punctures of arbitrary size.

have only 38 terms.

\section{Conclusion}

We have rigorously analysed the effect of punctures on the area law of polygon models. In particular we obtained expressions for the leading amplitudes of the area moments for punctured polygons in terms of the amplitudes for unpunctured polygons (see Theorems 1 and 2). For staircase polygons this led to exact formulas for the amplitudes. For selfavoiding polygons the formulas rely on an assumption about the asymptotic behaviour of SAPs without punctures. They contain constants not known exactly but estimated numerically to a very high degree of accuracy. Our analysis also led to conjectures about the scaling behaviour of these models (see Conjecture 1). A proof of these conjectures is an open and difficult problem. Further numerical support of these conjectures might follow from an analysis of critical perimeter moments along the lines of [15].

The expressions for the amplitudes were thoroughly checked numerically. For staircase polygons with up to 5 minimal punctures and staircase polygons with one or two punctures of fixed size we used our series expansions to find the exact generating functions for area moments to order 10. Naturally, in all these cases the leading amplitude agrees with the proved formulas. Interestingly we find that the amplitude of the correction term is proportional to the corresponding leading amplitude with one less puncture. For staircase polygons with one and two punctures of arbitrary size a careful asymptotic analysis of the series for the area moments yielded very accurate estimates for the amplitudes, again confirming the exact formulas. Finally, we also analysed series for self-avoiding polygons with one and two punctures (minimal as well as arbitrary). In this case the numerical 
evidence is not quite as convincing, but we did find that the numerical estimates agree with the exact formulas to at least 3-4 significant digits.

The numerical analysis also yielded explicit expressions for correction terms, see Eqs. (28), (34) and subsection 6.2. These correspond to contributions from punctureboundary interactions and from puncture-puncture interactions. It would be interesting, but difficult to give a combinatorial proof of these results. The difficulty of any combinatorial proof becomes clearer when considering the recent closed form solution of a closely related model of punctured staircase polygons. In [43] one of us (IJ) considered a model of punctured staircase polygons in which the internal polygon is rotated by 90 degrees with respect to the outer polygon. The proofs in this paper never consider such restrictions on the placement of the internal polygon, that is, the internal polygon can be placed in any way one pleases. The results therefore carry over unaltered to the problem of rotated punctured staircase polygons. Interestingly, this means that the leading asymptotic form of the coefficients is exactly the same for both models, any differences arising only from the correction terms. The dominant correction term to the half-perimeter generating function for punctured staircase polygons $\mathcal{P}(x)$ is $\propto \log (1-4 x) / \sqrt{1-4 x}$ [22]. From the exact closed form solution to the half-perimeter generating function for rotated punctured staircase polygons $\mathcal{P}_{\text {Rot }}(x)$ it follows that the first correction term is $\propto(1-4 x)^{-3 / 4}$ [43]. These differences indicate that combinatorial arguments for a proof of sub-dominant behaviour must be quite subtle!

In this paper, we discussed models of punctured polygons, where punctures are of the same type as the outer polygon. More generally, punctures may be built from different polygon classes. In that situation, Theorem 1 holds, with the obvious modification, for any collection of polygon models as punctures. Theorem 2 holds, with the obvious modification, for any collection of polygon models as punctures, if the corresponding half-perimeter generating functions are finite at the radius of convergence $x_{c}$ of the halfperimeter generating function of the outer polygon. This includes the model considered in [43] as a special case. We remark that the critical half-perimeter generating function of the outer polygon may be infinite.

Our analytical results are based on the observation that puncture counting can be done using polygon area estimates, polygon boundary contributions being asymptotically negligible. In particular, effects of self-avoidance do not influence the results. This phenomenon is also expected to hold in higher dimension. Consider so-called polycubes, the generalisation of polyominoes to three dimensions. Polycubes have been enumerated by volume up to 18 cubes, see [44]. Three-dimensional vesicles [45] are a subclass of polycubes, having no interior holes. Let a class of three-dimensional vesicles be given, counted by surface area, with a bounded number of vesicular holes. Assume that the asymptotic behaviour of the volume moments is known for the model without vesicular holes. If $0<\phi<1$ and the critical surface area generating function is finite, then our method of proof can be adapted to describe the volume law of three-dimensional vesicles with vesicular holes. For the full model of closed self-avoiding orientable surfaces of genus zero on the cubic lattice, however, there is numerical evidence that $\phi=1$, see e.g. the review in $[3]$. 
For models with minimal punctures, the number $p_{m}^{\square(1,0)}$ in Eq. (15) also counts the number of polygons winding around a fixed point of the dual lattice. This problem and its generalisation to $M>1$ mutually avoiding self-avoiding polygons has been considered previously by Cardy [46]. It would be interesting to consider whether this generalisation can also be treated using the above methods. This involves the analysis of polygon models satisfying $\theta=0$. If $\theta<0$, interaction terms are generally not asymptotically negligible, so that the above analysis does not yield asymptotically exact estimates.

A major open question is the problem of self-avoiding polygons with an unbounded number of punctures. Here, Theorem 2 yields an upper bound. Let $\mathcal{Q}_{k}(x)$ denote the $k^{t h}$ area moment generating function for a model of punctured polygons with an arbitrary number of punctures. $\mathcal{Q}_{k}(x)$ is a formal power series, usually with zero radius of convergence. The (asymptotically exact) upper bound for models with $r$ punctures yields an upper estimate for the number of punctured polygons with an arbitrary number of punctures. It is

$$
\mathcal{Q}_{k}(x) \ll \sum_{r=0}^{\infty} \frac{\mathcal{P}_{k+r}(x)}{r !}\left(\mathcal{P}_{0}(x)\right)^{r}
$$

where $\ll$ denotes coefficientwise majorisation. Let $\widetilde{\mathcal{Q}}_{k}(x)$ denote the $k^{\text {th }}$ factorial area moment generating function for punctured polygons with an arbitrary number of punctures. It can be shown that an upper bound is given by

$$
\widetilde{\mathcal{Q}}_{k}(x) \ll \sum_{r=0}^{\infty} \frac{\widetilde{\mathcal{P}}_{k+r}(x)}{r !}\left(\widetilde{\mathcal{P}}_{0}(x)\right)^{r}=\left.\frac{\mathrm{d}^{k}}{\mathrm{~d} q^{k}} \mathcal{P}(x, q)\right|_{q=1+\mathcal{P}(x, 1)},
$$

where $\widetilde{\mathcal{P}}_{k}(x)$ is the $k^{\text {th }}$ factorial moment generating function, and $\mathcal{P}(x, q)$ is the perimeter and area generating function of the model without punctures. In particular, the halfperimeter generating function $\mathcal{Q}_{0}(x)=\widetilde{\mathcal{Q}}_{0}(x)$ is majorised by

$$
\mathcal{Q}_{0}(x) \ll \mathcal{P}(x, 1+\mathcal{P}(x, 1)) .
$$

Another problem touched upon in this article is punctured polygons in the fixed area ensemble. We gave a (non-rigorous) argument for values of the critical exponent in the branched polymer phase from the crossover behaviour of the tentative scaling function, thereby confirming previous results [21]. In that phase, boundary effects are indeed crucial, such that our methods of deriving limit distributions cannot be applied in this situation. On the other hand, area laws are expected to be of Gaussian type, as is usually the case away from phase transition points. The same phenomenon is expected to occur in the fixed perimeter ensembles for $q \neq 1$.

\section{E-mail or WWW retrieval of series}

The series for the generating functions studied in this paper can be obtained via e-mail by sending a request to I.Jensen@ms.unimelb.edu.au or via the world wide web on the URL http://www.ms.unimelb.edu.au/ iwan/ by following the instructions. 


\section{Acknowledgements}

The calculations presented in this paper would not have been possible without a generous grant of computer time on the server cluster of the Australian Partnership for Advanced Computing (APAC). We also used the computational resources of the Victorian Partnership for Advanced Computing (VPAC). CR and AJG would like to acknowledge support by the German Research Council (Deutsche Forschungsgemeinschaft) within the CRC701. IJ and AJG gratefully acknowledge financial support from the Australian Research Council.

\section{Appendix}

We analyse the asymptotic growth of a Cauchy product of sequences in terms of the asymptotic growth of its constituting sequences. The following lemma is an extension of [47, Thm 2] to the case of generating functions with equal radii of convergence. Its proof relies on Lebesgue's dominated convergence theorem [48, Thm 1.34], which states conditions under which an exchange of limit and sum is allowed: With $n \in \mathbb{N}$ and $k \in \mathbb{N}_{0}$ let real numbers $a_{n, k}$ be given. Assume that $\lim _{n \rightarrow \infty} a_{n, k}=: a_{k} \in \mathbb{R}$ for all $k$ and that for all $k$ there is a bound $\left|a_{n, k}\right| \leq b_{k}$ uniformly in $n \in \mathbb{N}$. Assume that $\sum_{k>0} b_{k} \in \mathbb{R}$. Then $\lim _{n \rightarrow \infty} \sum_{k \geq 0} a_{n, k}=\sum_{k \geq 0} a_{k} \in \mathbb{R}$.

Lemma 1. Let two sequences $\left(f_{n}\right)_{n \in \mathbb{N}_{0}}$ and $\left(g_{n}\right)_{n \in \mathbb{N}_{0}}$ of real numbers be given, with generating functions $f(x)=\sum_{n \geq 0} f_{n} x^{n}$ and $g(x)=\sum_{n \geq 0} g_{n} x^{n}$. Assume that both generating functions have the same positive finite radius of convergence $x_{c}, 0<x_{c}<\infty$. Assume that the sequences $\left(f_{n}\right)$ and $\left(g_{n}\right)$ satisfy asymptotically

$$
f_{n} \sim A x_{c}^{-n} n^{\gamma-1}, \quad g_{n} \sim B x_{c}^{-n} n^{\delta-1} \quad(n \rightarrow \infty),
$$

for nonzero numbers $A \neq 0, B \neq 0$, and for real constants $\gamma, \delta$ satisfying $\delta<0$ and $\gamma>\delta+1$. Assume that $g\left(x_{c}\right):=\lim _{x / x_{c}} g(x) \neq 0$. Then, the Cauchy product of $\left(f_{n}\right)$ and $\left(g_{n}\right)$ satisfies

$$
\sum_{k=0}^{n} f_{n-k} g_{k}=\left[x^{n}\right] f(x) g(x) \sim g\left(x_{c}\right) f_{n} \quad(n \rightarrow \infty) .
$$

Proof. Let $f_{n}:=0$ for $n<0$ and define for $n \in \mathbb{N}$ and for $k \in \mathbb{N}_{0}$

$$
a_{n, k}:=\frac{f_{n-k}}{A x_{c}^{-n} n^{\gamma-1}} g_{k} .
$$

Below, we derive a bound on $\left|a_{n, k}\right|$ uniformly in $n \in \mathbb{N}$ and summable in $k \in \mathbb{N}_{0}$. Then, Lebesgue's dominated convergence theorem can be applied to $a_{n, k}$. Since $g\left(x_{c}\right) \neq 0$, this yields the statement of the lemma.

Note first that the assumption on the asymptotic behaviour of $f_{n}$ implies the existence of a constant $n_{0} \in \mathbb{N}$ such that for all $n \geq n_{0}+k$ we have the estimate

$$
\left|\frac{f_{n-k}}{A x_{c}^{-n} n^{\gamma-1}}\right| \leq 2 x_{c}^{k}\left(1-\frac{k}{n}\right)^{\gamma-1} \text {. }
$$


Fix such $n_{0}$. We distinguish the three cases $n<k, k \leq n<n_{0}+k$, and $n \geq n_{0}+k$. If $n<k$, we clearly have $a_{n, k}=0$. For $n \geq n_{0}+k$, the above estimate yields

$$
\left|a_{n, k}\right|=\left|\frac{f_{n-k}}{A x_{c}^{-n} n^{\gamma-1}} g_{k}\right| \leq 2\left(1-\frac{k}{n}\right)^{\gamma-1} x_{c}^{k}\left|g_{k}\right| .
$$

We will first consider the case $\gamma-1<0$. If $n \geq n_{0}+k$, we have $1 /(1-k / n) \leq 1+k / n_{0}$. This implies

$$
\left|a_{n, k}\right| \leq 2^{2-\gamma_{1}} k^{1-\gamma} x_{c}^{k}\left|g_{k}\right|=: b_{k}^{(1)}
$$

If $k \leq n<n_{0}+k$, we estimate similarly

$$
\begin{aligned}
& \left|a_{n, k}\right|=\left|\frac{f_{n-k}}{A x_{c}^{-n} n^{\gamma-1}} g_{k}\right| \leq|A|^{-1} \max \left\{1, x_{c}^{n_{0}}\right\} \max _{m<n_{0}}\left\{\left|f_{m}\right|\right\}\left(n_{0}+k\right)^{1-\gamma} x_{c}^{k}\left|g_{k}\right| \\
& \quad \leq|A|^{-1} \max \left\{1, x_{c}^{n_{0}}\right\} \max _{m<n_{0}}\left\{\left|f_{m}\right|\right\} \cdot\left\{\begin{array}{cc}
n_{0}^{1-\gamma}\left|g_{0}\right|, & k=0 \\
\left(2 n_{0}\right)^{1-\gamma} x_{c}^{k} k^{1-\gamma}\left|g_{k}\right|, & k \neq 0
\end{array}\right\}=: b_{k}^{(2)} .
\end{aligned}
$$

Define $b_{k}:=b_{k}^{(1)}+b_{k}^{(2)}$ for $k \in \mathbb{N}_{0}$. Then $\left|a_{n, k}\right| \leq b_{k}$ uniformly in $n \in \mathbb{N}$, and $\sum_{k \geq 0} b_{k}<\infty$. Summability follows from

$$
\sum_{k=0}^{\infty} x_{c}^{k} k^{1-\gamma}\left|g_{k}\right|<\infty
$$

since by assumption $x_{c}^{k} k^{1-\gamma}\left|g_{k}\right| \sim|B| k^{\delta-\gamma}$ as $k \rightarrow \infty$, where $\gamma-\delta>1$.

If $\gamma-1 \geq 0$, uniform estimates are obtained along the same lines. In that situation, the factors $(1-k / n)^{\gamma-1}$ and $\left(n_{0}+k\right)^{1-\gamma}$ may be replaced by unity, resulting in simpler uniform bounds involving $\sum_{k \geq 0} x_{c}^{k}\left|g_{k}\right|<\infty$.

\section{References}

[1] Madras N and Slade G 1993 The Self-Avoiding Walk (Boston: Birkhäuser)

[2] Hughes B D 1995 Random Walks and Random Environments, Vol I Random Walks (Oxford: Clarendon)

[3] Vanderzande C 1998 Lattice Models of Polymers Cambridge Lecture Notes in Physics 11 (Cambridge: Cambridge University Press)

[4] de Gennes P G 1979 Scaling Concepts in Polymer Physics (Ithaca: Cornell University Press)

[5] Leibler S, Singh R R P and Fisher M E 1987 Thermodynamic behavior of twodimensional vesicles Phys. Rev. Lett. 59 1989-1992

[6] Gutman I and Cyvin S 1989 Introduction to the Theory of Benzenoid Hydrocarbons (Berlin: Springer)

[7] Vöge M, Guttmann A J and Jensen I 2002 On the number of benzenoid hydrocarbons, J. Chem. Inf. Comput. Sci. 42 456-66 
[8] Fisher M E, Guttmann A J and Whittington S G 1991, Two-dimensional lattice vesicles and polygons, J. Phys. A: Math. Gen. 24, 3095-3106

[9] Nienhuis B 1982 Exact critical point and critical exponents of $\mathrm{O}(n)$ models in two dimensions Phys. Rev. Letts. 49 1062-65

[10] Nienhuis B 1984 Critical behavior of two-dimensional spin models and charge asymmetry in the coulomb gas J. Stat. Phys. 34 731-761

[11] Richard C, Guttmann A J and Jensen I 2001 Scaling function and universal amplitude combinations for self-avoiding polygons J. Phys. A: Math. Gen. 34 L495-501

[12] Cardy J 2001 Exact scaling functions for self-avoiding loops and branched polymers J. Phys. A: Math. Gen. 34 L665-L672

[13] Richard C 2002 Scaling behaviour of two-dimensional polygon models J. Stat. Phys. 108 459-493

[14] Richard C, Jensen I and Guttmann A J 2003 Scaling function for self-avoiding polygons in Proceedings of the International Congress on Theoretical Physics TH2002 (Paris), Supplement (eds Iagolnitzer D, Rivasseau V and Zinn-Justin J) (Basel: Birkhäuser) 267-277.

[15] Richard C, Jensen I and Guttmann A J 2004 Scaling function for self-avoiding polygons revisited J. Stat. Mech.: Th. Exp. P08007

[16] Guttmann A J, Jensen I, Wong L H and Enting I G 2000 Punctured polygons and polyominoes on the square lattice J. Phys. A: Math. Gen. 33 1735-1764

[17] Janse van Rensburg E J and Whittington S G 1989 Self-avoiding surfaces J. Phys. A: Math. Gen. 22 4939-4958

[18] Guttmann A J, Jensen I and Owczarek A L 2001 Polygonal polyominoes on the square lattice J. Phys. A: Math. Gen. 34 3721-3733

[19] Janse van Rensburg E J 1999 Models of composite polygons J. Phys. A: Math. Gen. 32 4351-4372

[20] Bleistein N and Handelsman Richard A 1986 Asymptotic Expansions of Integrals 2nd ed (New York: Dover)

[21] Janse van Rensburg E J 1992 Surfaces in the hypercubic lattice J. Phys. A: Math. Gen. 25 3529-3547

[22] Guttmann A J and Jensen I 2006 The perimeter generating function of punctured staircase polygons J. Phys. A: Math. Gen. 39 3871-3882

[23] Prellberg T and Owczarek A 1999 On the asymptotics of the finite-perimeter partition function of two-dimensional lattice vesicles Commun. Math. Phys. 201 493-505

[24] Janse van Rensburg E J 2000 The Statistical Mechanics of Interacting Walks, Polygons, Animals and Vesicles Oxford Lecture Series in Mathematics and its Applications 18 (Oxford: Oxford University Press)

[25] Flajolet P and Sedgewick R 2008 Analytic Combinatorics in press (Cambridge: Cambridge University Press) 
[26] Brydges D C and Imbrie J Z 2003 End-to-end distance from the Green's function for a hierarchical self-avoiding walk in four dimensions Commun. Math. Phys. 239 $523-547$

[27] Prellberg T 1994 Uniform q-series asymptotics for staircase polygons J. Phys. A: Math. Gen. 28 1289-1304

[28] Louchard G 1984 The Brownian excursion area: a numerical analysis Comput. Math. Appl. 10 413-417; Louchard G 1986 Erratum: "The Brownian excursion area: a numerical analysis" Comput. Math. Appl. 12375

[29] Takács L 1991 On a probability problem connected with railway traffic J. Appl. Math. Stoch. Anal. 4 1-27

[30] Flajolet P and Louchard G 2001 Analytic variations on the Airy distribution Algorithmica 31 361-377

[31] Chung K L 1974 A Course in Probability Theory (New York: Academic Press) 2nd ed

[32] Flajolet P 1999 Singularity analysis and asymptotics of Bernoulli sums Theoret. Comput. Sci. 215 371-381

[33] Richard C 2008 On $q$-functional equations and excursion moments Discrete Math., in press; math.CO/0503198

[34] Richard C 2006 Staircase polygons: moments of diagonal lengths and column heights J. Phys.: Conf. Ser. 42 239-257

[35] Bousquet-Mélou M 1996 A method for the enumeration of various classes of columnconvex polygons Discrete Math. 154 1-25

[36] Brak R, Owczarek A L and Prellberg T 1993 A scaling theory of the collapse transition in geometric cluster models of polymers and vesicles J. Phys. A: Math. Gen. 26 4565-4579

[37] Richard C and Guttmann A J 2001 q-linear approximants: scaling functions for polygon models J. Phys. A: Math. Gen. 34 4783-4796

[38] Jensen I 2003 A parallel algorithm for the enumeration of self-avoiding polygons on the square lattice J. Phys. A: Math. Gen. 36 5731-5745

[39] Jensen I 2000 Size and area of square lattice polygons J. Phys. A: Math. Gen. 33 3533-3543

[40] Guttmann A J 1989 Asymptotic analysis of power-series expansions in Phase Transitions and Critical Phenomena vol. 13 (eds Domb C and Lebowitz J L) (New York: Academic) 1-234

[41] Jensen I 2006 Honeycomb lattice polygons and walks as a test of series analysis techniques J. Phys.: Conf. Ser. 42 163-178.

[42] Jensen I and Guttmann A J 1999 Self-avoiding polygons on the square lattice J. Phys. A: Math. Gen. 32 4867-4876 
[43] Jensen I 2006 Exact perimeter generating function for a model of punctured staircase polygons Preprint: cond-mat/0610605

[44] Aleksandrowicz G and Barequet G 2006 Counting $d$-dimensional polycubes and nonrectangular planar polyominoes in: Proc. 12th Ann. Int. Computing and Combinatorics Conf. (COCOON), Taipei, Taiwan Springer Lecture Notes in Computer Science 4112 418-427

[45] Whittington S G 1993 Statistical mechanics of three-dimensional vesicles J. Math. Chem. 14 103-100

[46] Cardy J 2000 Linking numbers for self-avoiding loops and percolation: application to the spin quantum hall transition Phys. Rev. Lett. 84 3507-3510

[47] Bender E A 1974 Asymptotic methods in enumeration SIAM Rev. 16 485-515; Errata SIAM Rev. 18 (1976) 292

[48] Rudin W 1987 Real and Complex Analysis (New York: McGraw-Hill) 